\title{
Testing the black hole "no-hair" hypothesis
}

\author{
Vitor Cardoso ${ }^{\star, \dagger, \ddagger}$, Leonardo Gualtieri॰ \\ * CENTRA, Departamento de Física, Instituto Superior Técnico, Universidade de \\ Lisboa, Avenida Rovisco Pais 1, 1049 Lisboa, Portugal \\ $\dagger$ Perimeter Institute for Theoretical Physics, 31 Caroline Street North Waterloo, \\ Ontario N2L 2Y5, Canada \\ ¥ Theoretical Physics Department, CERN, CH-1211 Genève 23, Switzerland \\ - Dipartimento di Fisica, "Sapienza" Università di Roma \& Sezione INFN Roma 1, \\ P.A. Moro 5, 00185, Roma, Italy \\ E-mail: vitor.cardoso@ist.utl.pt, Leonardo.Gualtieri@roma1.infn.it
}

\begin{abstract}
Black holes in General Relativity are very simple objects. This property, that goes under the name of "no-hair," has been refined in the last few decades and admits several versions. The simplicity of black holes makes them ideal testbeds of fundamental physics and of General Relativity itself. Here we discuss the no-hair property of black holes, how it can be measured in the electromagnetic or gravitational window, and what it can possibly tell us about our universe.
\end{abstract}

\section{Contents}

1 What is the no-hair hypothesis (and why should we care)? 3

1.1 The simplicity of black holes in General Relativity . . . . . . . . . . 3

1.2 The uniqueness and no-hair theorem $(\mathrm{s}) \ldots \ldots \ldots \ldots$

1.3 The "no-hair" or "Kerr" hypothesis . . . . . . . . . . . . . 6 6

2 The dynamics of hair-loss for massless fields

3 The death of the no-hair hypothesis 9

3.1 Anisotropic fluid hair . . . . . . . . . . . . . . . . . . . 10

3.2 Hairy black holes in the Standard Model and extensions thereof . . . . . 11

3.3 Hairy black holes in other theories or frameworks . . . . . . . . 14

3.4 Horizonless compact objects . . . . . . . . . . . . . . . . 14

4 "No-hair" rises again 15

5 Tests of the "no-hair" hypothesis 16

5.1 Multipole moments . . . . . . . . . . . . . . . . . . . 16

5.1.1 Multipolar expansions in Newtonian gravity and in general relativity 17

5.1.2 The multipole moments of some astrophysical objects . . . . . . 20 
5.2 Dynamical tests with gravitational waves: ringdown . . . . . . . . . . 21

5.2.1 Using two or more modes to test the Kerr hypothesis . . . . . . . 21

5.2.2 Constraining alternative theories . . . . . . . . . . 26

5.2 .3 Environmental effects . . . . . . . . . . . . . . . 27

5.2.4 QNMs as a probe of the event horizon . . . . . . . . . . 27

5.3 Dynamical tests with gravitational waves: inspiral . . . . . . . . . . . 28

5.4 Tests with electromagnetic observations . . . . . . . . . . . . . . . . 29

5.4 Motion of stars . . . . . . . . . . . . . . . . . 29]

5.4 .2 Motion of pulsars . . . . . . . . . . . . . . . . 31

5.4 .3 Accretion disks . . . . . . . . . . . . . . . . 32

5.4 .4 Black hole "shadows" . . . . . . . . . . . . . . . . 33

6 Conclusions $\quad 36$

Appendix: Multipole moments in Newtonian gravity and in general relativity $\quad\left[\begin{array}{l}36 \\ \hline\end{array}\right.$

A1: Multipole moments in Newtonian gravity . . . . . . . . . . . . 37

A2: Multipole moments in general relativity: the Geroch-Hansen construction 38

A3: Multipole moments in general relativity: Thorne's expansion . . . . . . . 40 


\section{What is the no-hair hypothesis (and why should we care)?}

\subsection{The simplicity of black holes in General Relativity}

We celebrate this year the first direct detection of gravitational waves (GWs) and the first detection of a black hole (BH) binary, in its last stages of coalescence [1]. In this context, it is appropriate to also honor the centenary of the Schwarzschild solution, which describes any regular asymptotically flat, static and spherically symmetric vacuum spacetime in General Relativity (GR). In standard Schwarzschild coordinates, the solution reads

$$
d s^{2}=-c^{2}\left(1-\frac{2 G M}{c^{2} r}\right) d t^{2}+\left(1-\frac{2 G M}{c^{2} r}\right)^{-1} d r^{2}+r^{2} d \Omega^{2},
$$

where $G$ is Newton's constant and $c$ the speed of light. The most distinctive feature of the solution above is a coordinate singularity at $r=2 G M / c^{2}$, describing a null surface (the event horizon) which causally separates the inside and outside regions. For physical setups where (static, spherically symmetric) matter is present, the solution should be truncated at the radius of the object and merged smoothly with some interior solution. For pure vacuum spacetimes, it describes a BH. In other words, any static BH in the Universe which is non-spinning and that lives in approximately empty surroundings is described by the geometry (1.1).

The Schwarzschild solution is fully characterized by a single parameter, the total gravitational mass $M$. In this respect, it is not dissimilar from its Newtonian counterpart: a spherically symmetric, vacuum, static solution of Newton's gravity is also described by only a mass parameter. It turns out to be difficult to construct $\mathrm{BH}$ solutions described by more parameters. For example, let's try to "anchor" a weak, static massless spin-s field onto the Schwarzschild solution. For the sake of illustration we focus on minimally coupled scalars, vector fields described by Maxwell's theory and gravitational fluctuations within vacuum GR [2]. At a linearized level (i.e., keeping the background geometry fixed), these fields can be expanded in scalar, vector or tensor harmonic functions, parametrized by an integer number $l=0,1,2, \ldots$, which carry information on the angular dependence of the field. These fields are all described by the equation [2]

$$
\left(\left(1-\frac{2 G M}{c^{2} r}\right) \Psi^{\prime}\right)^{\prime}-\left(\frac{l(l+1)}{r^{2}}+\frac{2 G M\left(1-s^{2}\right)}{c^{2} r^{3}}\right) \Psi=0,
$$

where $\Psi$ denotes the field amplitude, $s=0,1,2$ for scalars, vectors and tensors, respectively, and primes stand for radial derivatives. One can now multiply the above equation by the complex conjugate $\Psi^{*}$, integrate from the horizon to infinity, and look for regular solutions of the above equation. We get, upon performing an integration by parts and dropping a boundary term (regular solutions evaluate it to zero),

$$
-\int_{\frac{2 G M}{c^{2}}}^{+\infty} d r\left(1-\frac{2 G M}{c^{2} r}\right)\left|\Psi^{\prime}\right|^{2}+\frac{l(l+1)}{r^{3}}\left(r+\frac{2 G M\left(1-s^{2}\right)}{c^{2} l(l+1)}\right)|\Psi|^{2}=0
$$


For any $l \geq s$ the integrand is negative-definite outside the horizon, and the only solution to the above equation is the trivial one, $\Psi=0$. For vector fields, there is a nontrivial solution $\Psi=$ const for $l=0$, describing a weakly charged BH. For tensors, the only non-trivial solutions (for which the integrand is not positive-definite) have $l=0,1$ and correspond to a slight change of mass and addition of small amount of angular momentum [3]. This simple exercise then leads one to the following conclusions:

(i) It is impossible to "anchor" non-interacting scalars (and turns out, also fermions) onto a Schwarzschild BH.

(ii) The Schwarzschild solution allows, in principle, for a generalization that includes (only) electric charge and rotation.

Note that the conclusions above are drawn in the context of GR; other theories would lead to different equations of motion that could (and do, sometimes) lead to other types of solutions. Thus, non-rotating BHs do not exhibit any "protuberance." John Wheeler and others summarized these results with the expression "black holes have no hair", where "hair" is a measure of the complexity of the gravitational field of the geometry. We will soon see that, within GR, BHs can rotate and be electromagnetically charged. Thus in fact BHs have a finite number of hairs, although the term "no-hair" is still loosely applied to these solutions as well. In general, as we show below, if one characterizes the geometry through its multipole moments, BHs have only a finite and small number of independent quantities that suffice to completely describe its multipolar structure.

\subsection{The uniqueness and no-hair theorem(s)}

In fact, a charged and rotating $\mathrm{BH}$ solution was discovered many decades after Schwarzschild's work, and is now known as the Kerr-Newman BH, carrying mass, electric charge and angular momentum [4, 5]. In addition, in a series of uniqueness theorems (see [6] for reviews), it was established,

Theorem 1: an isolated, stationary and regular BH in Einstein-Maxwell theory is described by the Kerr-Newman family.

In other words, the structure of asymptotically flat, stationary BHs of EinsteinMaxwell theory is completely determined by its global charges defined at infinity, in particular its mass, angular momentum and electric charge. Astrophysical BHs are thought to be neutral to a very good approximation, because of quantum discharge effects, electron-positron pair production and charge neutralization by astrophysical plasmas [11] I. Because of this, we will focus almost entirely on electrically neutral geometries. The neutral version of the Kerr-Newman solution is simply described by

$\ddagger$ These mechanisms are suppressed in theories of minicharged dark matter, when the charged particles have a charge-to-mass ratio much smaller than that of the electron, and for which BHs may be charged under a hidden $U(1)$ symmetry [12. 
the two-parameter Kerr metric [4], which in standard Boyer-Lindquist coordinates reads

$$
\begin{aligned}
d s^{2} & =-c^{2}\left(1-\frac{2 G M r}{c^{2} \Sigma}\right) d t^{2}+\frac{\Sigma}{\Delta} d r^{2}+\Sigma d \theta^{2}-\frac{4 G J r \sin ^{2} \theta}{c^{2} \Sigma} d t d \phi \\
& +\left[r^{2}+\frac{J^{2}}{M^{2} c^{2}}+\frac{2 G J^{2} r \sin ^{2} \theta}{M c^{4} \Sigma}\right] \sin ^{2} \theta d \phi^{2},
\end{aligned}
$$

where $\Sigma \equiv r^{2}+\frac{J^{2}}{M^{2} c^{2}} \cos ^{2} \theta, \Delta=r^{2}-\frac{2 G M r}{c^{2}}+\frac{J^{2}}{M^{2} c^{2}}$.

Note that Theorem 1 does not require axi-symmetry, it being a consequence of stationarity. Note also that Theorem 1 as well as all results discussed in the text, makes further analiticity assumptions, which we do not discuss here (but see Refs. [13, 14]). Due to these assumptions (which are required to infer axi-symmetry from stationarity), there are also those who advocate that the no-hair theorems are less general than commonly stated (see [15] and references therein).

From now on, we will use geometric units with $G=c=1$, and express the Kerr solution as above with $\Sigma \equiv r^{2}+a^{2} \cos ^{2} \theta, \Delta=r^{2}-2 M r+a^{2}$. This metric describes the gravitational field of a spinning $\mathrm{BH}$ of mass $M$ and angular momentum $J=a M$. The event horizon is located at $r_{+}=M+\sqrt{M^{2}-a^{2}}$, and the BH spin is bounded from above by $|a| \leq M$.

This uniqueness result is also an example of a no-hair theorem, and the horizon plays a crucial role in exclusion of "hair," as it prevents information traveling at finite speeds (other then that conveyed by conserved charges) to cross the horizon. This expectation, originally conjectured by Wheeler, was put on firmer ground in a series of works. For example, Hartle [16] and Teitelboim [17-19] showed that it is impossible to measure baryon or lepton numbers of BHs. In flat space there is a long-range $1 / r^{5}$ potential between two collections of matter arising from the exchange of neutrino pairs between them. But once one of the neutrinos crosses the horizon, a weak-interaction force of this kind ceases to exist. A Kerr BH has no exterior neutrino field with classical effects.

In fact, two strong results emerged regarding BHs in the presence of fundamental fields. The first concerns the existence of stationary BHs surrounded by stationary matter, in the form of fundamental fields without self-interactions $\S$.

Theorem 2: an isolated, stationary and regular BH in the Einstein-Klein-Gordon or Einstein-Proca $\|$ theory with a time-independent boson is described by the Kerr family $[21,24]$.

The fields can be also massless and complex, but the theorem requires them to inherit the spacetime symmetries, i.e., no time-dependence. Physically, time-dependent fields will scatter to infinity and/or enter the horizon and therefore break the assumed stationarity. A non-trivial time-dependence can be excluded for real scalars,

$\S$ see Section 3 for counter-examples involving other forms of matter like anisotropic fluids.

|| The Einstein Proca theory describes a theory with a massive photon, see e.g., Ref. [20]. 
Theorem 3: an isolated, stationary and regular BH in the Einstein-Klein-Gordon theory with one real scalar is described by the Kerr family [21 24].

Real scalars with a nontrivial time dependence will give rise to a nontrivial quadrupole moment, therefore emitting GWs, again breaking the stationarity assumption. Notice that complex scalars are able to avoid this result, by producing a time-independent stress-energy tensor. At the same time, they are also able to avoid being absorbed at the horizon through a superradiant mechanism. We will see in Section 3.2 that in fact complex fields are able to produce hairy BHs.

We should mention that there are also some "no-hair" results for dynamical BH systems, and that these may even include evolving $\mathrm{BH}$ binaries. We refer the reader to Ref. [25] for a discussion of the general situation, but we will not discuss these examples any further here.

In summary, the "no-hair" theorem(s) are a set of proofs that - under some conditions - Kerr-Newman is the only possible asymptotically flat and regular solution of the field equations in the presence of fundamental fields (see Refs. [8, 26 29] for further details).

\subsection{The "no-hair" or "Kerr" hypothesis}

However, there is no proof that Kerr-Newman is the most generic solution of the field equations (see below in Section 3 for the reasons why such proof cannot exist). The "no-hair" or "Kerr" hypothesis states simply that the Kerr geometry - which depends on only two parameters - describes any $\mathrm{BH}$ in the universe (with the exception of those involved in highly-dynamical phenomena). This is no modest proposal!

One thus is led to two questions:

a. If one sets up some arbitrary initial conditions how is the final, Kerr state approached?

b. Is Kerr inevitably the final state?

c. How can one test for a. or b.?

We will try to answer these questions below. The no-hair hypothesis is pivotal to interpret observations of massive astrophysical bodies. The only other compact object that is agreed to populate the universe are neutron stars. But neutron stars in GR cannot be more massive than $\sim 3 M_{\odot}$ [30], as not even degeneracy pressure can be sustained for stars more massive than this limit. Thus, within the framework of GR with a standard matter sector, and of the no-hair hypothesis, any compact object $\mathbb{T}$ with mass larger than $\sim 3 M_{\odot}$ is a Kerr BH. Conversely, any observation of a compact object with mass larger than $\sim 3 M_{\odot}$ and with metric different from the Kerr geometry would inevitably signal a departure from standard physics (either in the gravitational or in the matter sector). Therefore tests of strong-field gravity targeting BH systems aim at verifying the "Kerr hypothesis" in various ways.

ฯ By compact we mean compactnesses $2 G M / c^{2} R \gtrsim 10^{-3}-10^{-2}$. 


\section{The dynamics of hair-loss for massless fields}
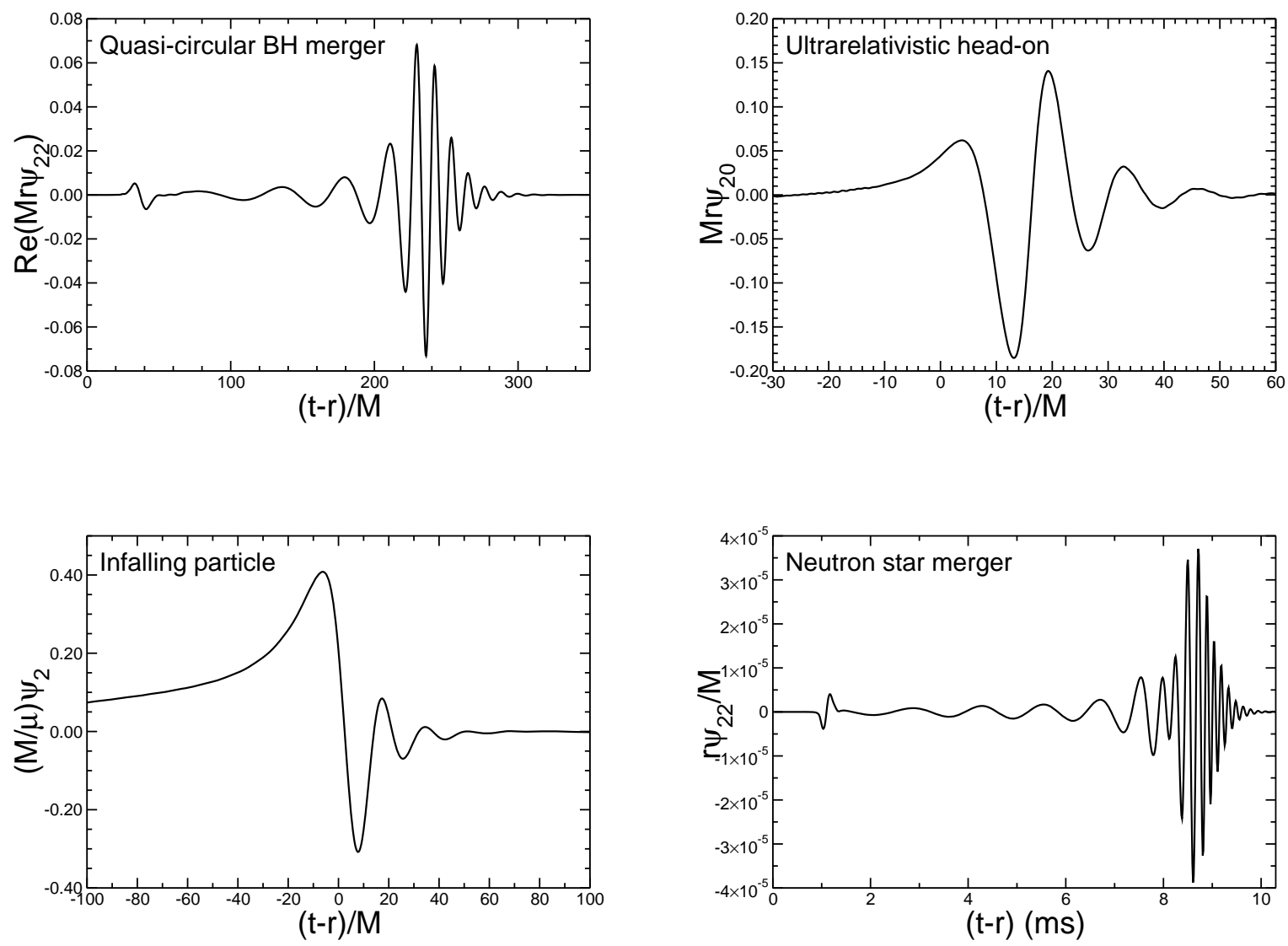

Figure 1. Four different physical processes leading to substantial quasinormal ringing. In all of them, quasinormal ringing is clearly visible. The upper-left panel (adapted from Ref. [31]) is the signal from two equal-mass BHs initially on quasi-circular orbits, inspiralling towards each other due to the energy loss induced by GW emission, merging and forming a single final BH. The upper-right panel shows gravitational waveforms from numerical simulations of two equal-mass BHs, colliding head-on with $v / c=0.94$ in the center-of-mass frame: as the center-of-mass energy grows (i.e., as the speed of the colliding BHs tends to the speed of light) the waveform is more and more strongly ringdown-dominated [32]. The bottom-left panel shows the gravitational waveform (or more precisely, the dominant, $l=2$ multipole of the Zerilli function) produced by a test particle of mass $\mu$ falling from rest into a Schwarzschild $\mathrm{BH}$ [33] : the shape of the initial precursor depends on the details of the infall, but the subsequent burst of radiation and the final ringdown are universal features. The bottom-right panel (reproduced from Ref. [34]) shows GWs emitted by two massive neutron stars with a polytropic equation of state, inspiralling and eventually collapsing to form a single $\mathrm{BH}$. With the exception of the infalling-particle case (where $M$ is the $\mathrm{BH}$ mass, $\mu$ the particle's mass and $\psi_{2}$ the Zerilli wavefunction), $\psi_{22}$ is the $l=m=2$ multipolar component of the Weyl scalar $\Psi_{4}, M$ denotes the total mass of the system and $r$ the extraction radius (see e.g. Ref. [31]). Taken from Ref. [2]. 
The first studies concerning the dynamics of BH spacetimes were mostly focused on Kerr BH backgrounds, weakly disturbed by massless, minimally coupled and noninteracting fields (see Section 3 below for the full picture). They included the scattering of Gaussian wavepackets [35] and the infall of a point-like particle [33]. These attempts showed that the GW (or scalar or electromagnetic) signal from a perturbed BH can in general be divided in three parts [2] (see Fig. 1). The signal starts with a prompt response at early times, which depends on the initial conditions and corresponds to direct propagation of the wave from source to observer.

After the prompt response, and as the source crosses the $\mathrm{BH}$ light ring (i.e, the unstable circular null geodesic), it excites the oscillation modes of the $\mathrm{BH}+$ The vibration modes of BHs are called quasinormal modes (QNMs) and consist on a superposition of exponentially damped sinusoids,

$$
\Psi \sim \sum_{l m n} A_{l m n} e^{i \omega_{l m n} t+\phi_{l m n}} e^{-t / \tau_{l m n}}=\sum_{l m n} A_{l m n} e^{2 \pi i f_{l m n} t+\phi_{l m n}} e^{-t / \tau_{l m n}},
$$

with characteristic QNM frequencies $\left(\omega_{l m n}, 1 / \tau_{l m n}\right)$ that depend only on the BH mass and spin, because the "progenitor" is fully characterized by these two parameters. These frequencies are tabulated and publicly available [2, 37, 38]. The modes depend on the integers $l, m$ labeling the angular dependence, where $l=2,3, \ldots$ and $m=0, \pm 1, \pm 2 \ldots \pm l$. The overtone index $n=0,1, \ldots[2,38]$.

For a Schwarzschild BH, the quadrupolar fundamental frequencies read

$$
\begin{aligned}
& f_{220}=f_{200}=1.207 \times 10^{-2}\left(\frac{10^{6} M_{\odot}}{M}\right) \mathrm{Hz}, \\
& \tau_{220}=\tau_{200}=55.37\left(\frac{M}{10^{6} M_{\odot}}\right) \mathrm{sec} .
\end{aligned}
$$

It is sometimes more convenient to work instead with the quality factor $Q_{l m n}=\omega_{l m n} \tau / 2$, a measure of how many ringdown cycles are contained in the signal. The frequencies and quality factor of Kerr BHs are shown in Fig. 2, as function of the dimensionless angular momentum $j \equiv a / M$. Highly spinning Kerr BHs are good resonators, the quality factor becoming very large.

In Refs. [2, 37, 38, the frequencies and quality factors of the first three overtones (for $l=2,3,4$ and all values of $m$ ) were fitted by functions of the form

$$
\begin{aligned}
& 2 \pi M f_{l m n}=f_{1}+f_{2}(1-j)^{f_{3}}, \\
& Q_{l m n}=q_{1}+q_{2}(1-j)^{q_{3}} .
\end{aligned}
$$

Here the constants $f_{i}$ and $q_{i}$ depend on $(l, m, n)$ (see Tables VIII-X in [38]), and the fits are accurate to better than $4 \%$ for $j \in[0,0.99]$.

Finally, at very late times, a power-law falloff of the field, not visible in Fig. 1. ensues [39, 40],

$$
\Psi \sim t^{-(2 l+3)} .
$$

This decay is clearly visible in evolutions of Gaussian wavepackets, as shown in Fig. 3 .

+ These modes can, alternatively, be thought of as a slow leakage of waves trapped in the circular null geodesic [36. 

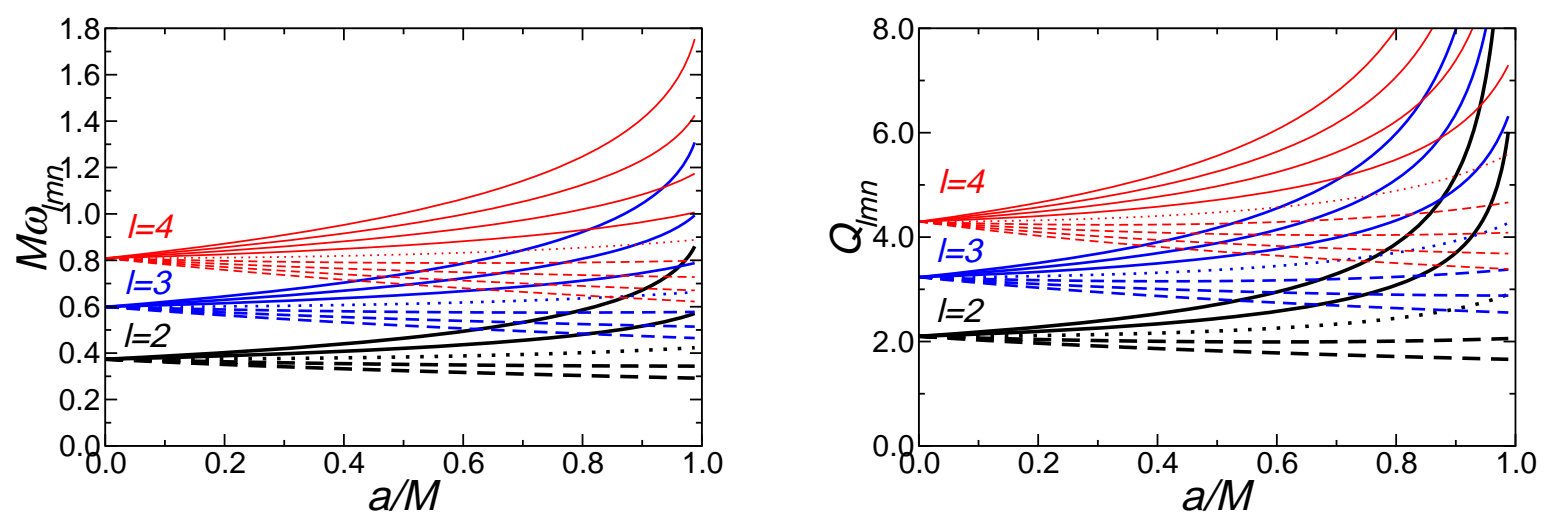

Figure 2. Frequencies and quality factors for the fundamental modes with $l=2,3,4$ and different values of $m$. Solid lines refer to $m=l, . ., 1$ (from top to bottom), the dotted line to $\mathrm{m}=0$, and dashed lines refer to $m=-1, . .,-l$ (from top to bottom). Quality factors for the higher overtones are lower than the ones we display here. Taken from Ref. [2].
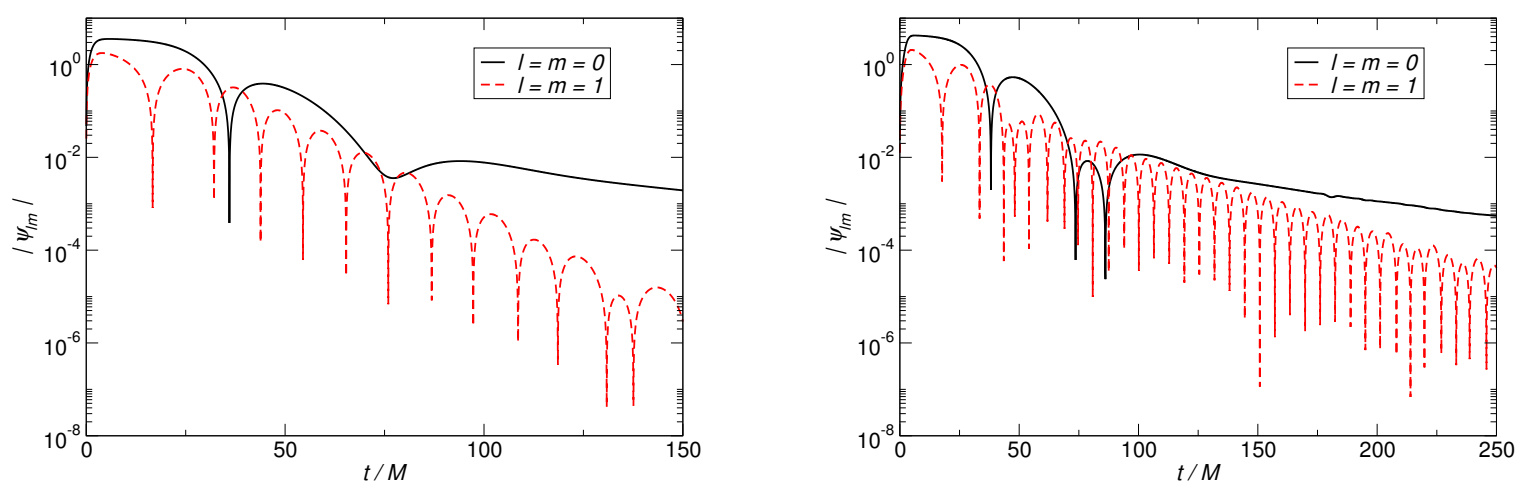

Figure 3. Evolution of a Gaussian profile of a massless scalar field with width $w=2 M$ centered at $r_{0}=12 M$ around a Schwarzschild (left panel) and a Kerr BH with $a / M=0.99$ (right panel). We depict the $l=m=0$ (solid black line) and $l=m=1$ (red dashed line) multipoles. The multipolar components of the field were extracted at $r_{\mathrm{ex}}=10$. The waveform displays an early transient followed by an exponentially decaying sinusoid (QNM ringdown) and a power-law tail at late times. The late-time power-law tail has the form $t^{p}$ for the monopole, with $p=-3.08$ for $a / M=0$ and $p=-3.07$ for $a / M=0.99$ in good agreement with the prediction $p=-3$ obtained from the low-frequency expansion of the wave equation (2.6). From Ref. 20].

\section{The death of the no-hair hypothesis}

Although very attractive, the no-hair hypothesis is dangerously close to either being (i) useless, if it requires vacuum or truly stationary spacetimes, in which case there is 
probably no $\mathrm{BH}$ in our universe satisfying these assumptions or (ii) contradicted by every-day observations of BHs surrounded by quasi-stationary matter, such as accretion disks or orbiting stars, since these systems are not described by the Kerr solution.

In other words, observations of BHs are bound to happen in "dirty" environments, not in vacuum. For massive BHs, it can be argued - and make the statement precise that accretion disks or orbiting stars can be disentangled from the BH background, by treating them as a small fluctuation. In this sense, tests of the no-hair hypothesis would still be possible, if the full spacetime deviates only "slightly" from that of a Kerr BH, and the "slightness" is controlled by the amount of matter that we see around the BH.

We will now argue, and show, that even at a fundamental level the "no-hair" hypothesis has been falsified several times in the past: there exist stationary solutions of the field equations which represent $\mathrm{BH}$ spacetimes that are not Kerr and that cannot be mapped to Kerr with the help of some small, observationally controlled parameter.

\subsection{Anisotropic fluid hair}

One intuitively expects that stationary - or at least very long-lived - hairy BHs are possible if one encloses a small spherical BH at the center of a large spherical "wall", with pressure just enough to keep the wall static. In other words, we do not expect anything drastic to occur if a, say, $1 \mathrm{Kg}$ or $10^{-24} \mathrm{~mm} \mathrm{BH}$ is placed inside a big room.

This expectation stands up to scrutiny. Analytical solutions describing an infinitelythin, spherically symmetric shell surrounding a static BH were constructed (and shown to be stable in some regions of parameter space [41, 42]). They are described by the geometry

$$
\begin{aligned}
& d s^{2}=-\alpha\left(1-\frac{2 M_{-}}{r}\right) d t^{2}+\left(1-\frac{2 M_{-}}{r}\right)^{-1} d r^{2}+r^{2} d \Omega^{2}, \quad r<R \\
& d s^{2}=-\left(1-\frac{2 M_{+}}{r}\right) d t^{2}+\left(1-\frac{2 M_{+}}{r}\right)^{-1} d r^{2}+r^{2} d \Omega^{2}, \quad r>R
\end{aligned}
$$

with $\alpha=\frac{1-2 M_{+} / R}{1-2 M_{-} / R}$ and where $R$ stands for the radius of the shell, which is described by an energy surface density $\sigma$ and pressure $P$, related through

$$
k_{-}-k_{+}=4 \pi R \sigma, \quad \frac{M_{+}}{k_{+}}-\frac{M_{-}}{k_{-}}=4 \pi R^{2}(\sigma+2 P),
$$

with $k_{ \pm}=\left(1-2 M_{ \pm} / R\right)^{1 / 2}$. A finite-thickness shell version was studied recently [43, 44].

The previous solution is somewhat artificial, in that the shell of matter is infinitely thin, and supported outside the horizon. However, even reasonable "short-hair" ("short" because it can be localized arbitrarily close to the horizon) solutions are possible, and can be found in closed analytical form. An example of a $\mathrm{BH}$ solution surrounded by an anisotropic fluid is described by [45],

$$
\begin{aligned}
d s^{2} & =-f d t^{2}+\frac{d r^{2}}{f}+r^{2} d \theta^{2}+r^{2} \sin ^{2} \theta d \phi^{2} \\
f & =1-\frac{2 M}{r}+\frac{Q_{m}^{2 k}}{r^{2 k}}
\end{aligned}
$$




$$
\rho=\frac{Q_{m}^{2 k}(2 k-1)}{8 \pi r^{2 k+2}}, \quad P=k \rho
$$

where $\rho$ and $P$ are the density and (angular) pressure, respectively, of the anisotropic fluid, and $Q_{m}$ is a constant, describing the "matter-hair". The stress-energy tensor of the fluid is given by

$$
T_{\mu \nu}=\rho U_{\mu} U_{\nu}+P \sigma_{a b}-\rho u_{\mu} u_{\nu},
$$

where $U_{\mu}$ is the fluid four-velocity, $u_{\mu}$ is a unit radial vector such that $u^{\mu} U_{\mu}=0$ and $\sigma_{\mu \nu}=g_{\mu \nu}+U_{\mu} U_{\nu}-u_{\mu} u_{\nu}$. The solution above corresponds to an anisotropic stress-tensor, specified by (3.2) in Ref. [45], and it reduces to the Reissner-Nordstrom BH when $k=1$.

It seems unlikely that these hairy solutions form in practice, in any kind of realistic environment. However, hairy solutions that have been observed in numerical simulations are compact torii around the final $\mathrm{BH}$ formed as the endstate of the coalescence and merger of two neutron stars [46]. These torii can have masses as large as $10 \%$ of the total mass, and seem to be stable on dynamical timescales.

\subsection{Hairy black holes in the Standard Model and extensions thereof}

The above solutions refer to fluids, which are themselves effective descriptions of fermions, and have not - with the exception of torii - been observed to form in realistic scenarios. We will now discuss a more natural framework for hairy BHs, in the context of fundamental fields. We will not try to make a systematic classification of solutions in this setup, and instead refer the reader to some of the outstanding reviews out there [8, 26-29, 47]. We would like to stress however, that all solutions need massive or self-interacting fields, in line with the fluid counterpart discussed previously.

Conceptually, the discovery of BHs with "color" (static BH solutions in EinsteinYang Mills (EYM) theory [48, 49], that require for their complete specification an additional parameter - besides the mass - not associated with any conserved charge), was the first example showing that the no-hair hypothesis needs revision. The theory is EYM with $\mathrm{SU}(2)$ gauge group, described by the action

$$
S=\int d^{4} x \sqrt{-g}\left(R / 16 \pi G-F_{\mu \nu}^{a} F^{a \mu \nu} / g^{2}\right),
$$

where $F_{\mu \nu}^{a}$ is the Yang-Mills field strength and $1 / g^{2}$ the coupling constant. To see how new solutions are possible, let's go back to the perturbative framework of Section 1.1 and "freeze" gravity by sending $1 / g^{2} \rightarrow 0$. Then, gravity effectively decouples from the YM field, and any vacuum solution solves the Einstein equations. Let us take again a Schwarzschild background. The YM field can be expressed in terms of a field $\Psi(t, r)=\psi(r) e^{-i \omega t}$. The radial wavefunction $\psi(r)$ satisfies the equation [50, 51]

$$
f\left(f \psi^{\prime}\right)^{\prime}+\left(\omega^{2}+f \frac{1-\psi^{2}}{r^{2}}\right) \psi=0,
$$

with $f=1-2 M / r$. Note that trivial static solutions to this equation are $\psi=0,1$. Equation (3.5) is stable on the $\psi=1$ branch: its linearized (around $\psi=1$ ) version 


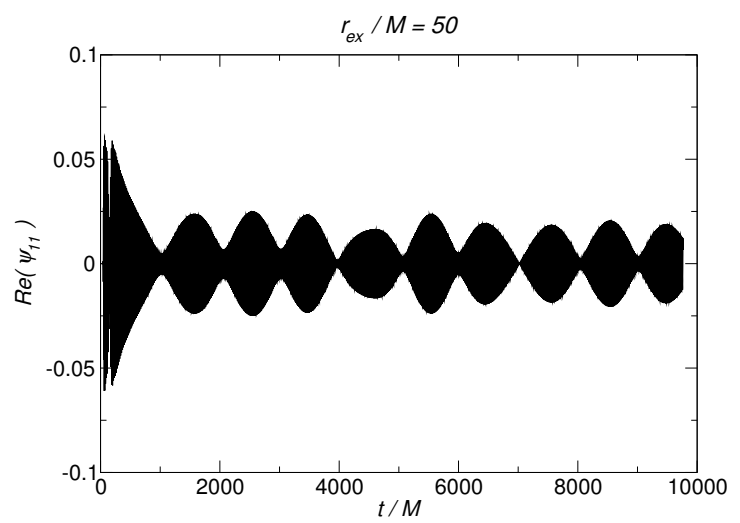

Figure 4. Evolution of (the dipole component of) a scalar Gaussian wavepacket in a background Kerr geometry, with $a=0.99 M$. The scalar has mass parameter $M \mu_{S}=0.42$. From Ref. 20].

is identical to that of $l=1$ electromagnetic modes, and a fundamental frequency $M \omega=0.248263-i 0.0924877$ satisfies the necessary boundary conditions [51]. On the other hand, linearization around $\Psi=0$ yields unstable solutions, as is easy to prove. We find the unstable mode $M \omega=i 0.1232877$. This indicates that there is a nontrivial static $(\omega=0)$ solution of equation (3.5). Such solution can be found imposing the appropriate asymptotic behavior at the horizon and demanding that $\psi(\infty)=1$.

The violation of the no-hair hypothesis was confirmed with the discovery of static BH solutions in theories like Einstein-Skyrme [52 54], and Einstein-non Abelian-Proca [55, 56].

As Bekenstein pointed out, these hairy solutions are possible in a way that parallels the existence of charged BHs: the gauge invariance of electrodynamics causes the Coulomb potential to propagate instantaneously in an appropriate gauge. Thus, the argument that information about hair cannot leave the horizon because it would need to travel faster than light, does not apply to the Coulomb potential, and charged BHs exist. Likewise, it seems intuitive that gauge invariance of non-abelian gauge theories should allow one or more of the gauge field components generated by sources in a $\mathrm{BH}$ to "escape" from it [6].

It is possible that a different mechanism might act to allow hairy BHs to exist: instead of allowing the hair to leave the horizon, one could in principle prevent it from falling in, in the first place! Superradiance in BH physics allows for precisely this, amplifying low-frequency bosonic waves, at the expense of the "horizon's rotational energy" [57. For massive fields, a finite-height barrier exists at large distances, confining the field and triggering an instability. Thus Kerr BHs are unstable against massive bosonic perturbations [57 61]. To be specific, the theory of a minimally coupled scalar 
with a mass term $\mu_{S}$

$$
S=\int d^{4} x \sqrt{-g}\left(\frac{R}{\kappa}-\frac{1}{2} g^{\mu \nu} \bar{\Psi}_{, \mu} \Psi_{, \nu}-\frac{\mu_{S}^{2} \bar{\Psi} \Psi}{2}\right) .
$$

admits Kerr BHs solutions which are linearly unstable against fluctuations of the scalar $\Psi$. The nonlinear development of the instability is not yet known [20, 62, 63], but linearized evolutions show that sufficiently small dimensionless coupling $M \mu_{S}$ leads to configurations that can live longer than a Hubble time around a Kerr BH. An example of the evolution of a Gaussian wavepacket around a Kerr BH is shown in Fig. 4. Notice how differently massless and massive fields behave (compare with Fig. 3). As such, even minimally coupled massive scalars produce what for all purposes are hairy solutions.

It seems intuitive that for real fields, the instability acts in such a way as to reduce the angular momentum off the BH, producing a slowly-spinning Kerr BH [64]. This is also a consequence of Theorem 3 in Section 1.2. Complex fields on the other hand, may give rise to a time-independent stress-energy tensor and therefore avoid energy loss, and both Theorem 1 and Theorem 2. It is thus not surprising that new solutions might branch off Kerr. These solutions were described in Refs. [28, 65] and are the perfect (because of their simplicity) example of how no-hair theorems can be circumvented. These "hairy" solutions can be generalized to complex vectors [66] (for which the Kerr geometry is also linearly unstable [20, 57, 67, 68]) and also to self-interacting scalars 69, 70].

Models of minicharged dark matter predict the existence of new fermions which possess a fractional electric charge or are charged under a hidden $U(1)$ symmetry [7176]. Their corresponding charge is naturally much smaller than the electron charge and their coupling to the Maxwell sector is suppressed. The following classical Lagrangian captures these theories 73

$$
\mathcal{L}=\sqrt{-g}\left(\frac{R}{16 \pi}-\frac{1}{4} F_{\mu \nu} F^{\mu \nu}-\frac{1}{4} B_{\mu \nu} B^{\mu \nu}+4 \pi e j_{\mathrm{em}}^{\mu} A_{\mu}+4 \pi e_{h} j_{h}^{\mu} B_{\mu}+4 \pi \epsilon e j_{h}^{\mu} A_{\mu}\right)
$$

where $F_{\mu \nu}:=\partial_{\mu} A_{\nu}-\partial_{\nu} A_{\mu}$ and $B_{\mu \nu}:=\partial_{\mu} B_{\nu}-\partial_{\nu} B_{\mu}$ are the field strengths of the ordinary photon and of the dark photon, respectively, $j_{\mathrm{em}}^{\mu}$ and $j_{h}^{\mu}$ are the electromagnetic and the hidden number currents, $e$ is the electron charge, and $e_{h}$ is the gauge coupling of the hidden sector. The model (3.7) describes a theory in which a charged fermion is coupled to ordinary photons with coupling $\epsilon^{2} e^{2}$ and to dark photons with coupling $e_{h}^{2}:=\epsilon_{h}^{2} e^{2}$. The parameters $\epsilon$ and $\epsilon_{h}$ are free. In this theory, BHs are described by the Kerr-Newman family of geometries, but now the classical and quantum discharge mechanisms can be suppressed. Thus, BHs can acquire electric "hair" [12].

Finally, another possible mechanism for hair growth, consists on dropping the stationarity assumption, and use time-dependent boundary conditions. Under certain conditions, this can lead to non-trivial BH geometries [25, 77, 78]. 


\subsection{Hairy black holes in other theories or frameworks}

In addition to simple extensions of GR, there are a number of proposed modifications of GR that actually change the gravity sector. The "zoo" is too big to describe here, we refer the reader to recent reviews on the topic [27, 79]. Some modifications are stringtheory motivated and are also expected to arise in a completion of GR, and in this sense it is not too surprising to find theories which include higher-order-in-curvature terms, like Chern-Simons gravity [80] or Einstein-dilaton Gauss-Bonnet (EDGB) gravity [81, 82]. These theories give rise to hairy $\mathrm{BH}$ solutions $[81$ 85]. We should also point out that even scalar-tensor theories can give rise to BHs surrounded by scalar fields, although the scalar fields needs nontrivial matter content to be anchored on [86, 87].

Other modifications of GR are motivated by a search for massive gravitons, a quest related to solutions of the cosmological constant problem, but also to nonlinear completions of GR. BHs in some of these theories also circumvent uniqueness and nohair results and may be surrounded by massive-graviton hair [29, 88, 89]. Hairy BH solutions also appear in Lorentz-violating gravity theories. BH solutions naturally have hair in these theories, because the preferred foliation can be described in terms of a scalar field (see e.g. [90] and references therein).

Recent studies of quantum effects in $\mathrm{BH}$ geometries argue that BHs should be surrounded by "soft" hair at quantum level [91], while some advocate that hair is a rule rather than the exception [92].

To conclude, BHs acquire hair in a variety of setups.

\subsection{Horizonless compact objects}

The no-hair hypothesis in astrophysical settings is tangled with the assumption that the compact object is too massive and compact to be anything else than a $\mathrm{BH}$. Theories as simple as gravitating massive scalars or vectors give rise to objects - boson stars and oscillatons - which could mimic BHs: they can be very massive, they are dark because the interaction cross-section with normal matter is small, and they can be very compact [93 98. It turns out that even the optical appearance of boson stars can be similar to that of $\mathrm{BHs}$ [99. Other proposals for compact massive objects include gravastars [100, superspinars [101], wormholes [102] and even mixed wormhole-star systems [103. Superspinars invoke the existence of unknown quantum effects that allow the existence of Kerr geometries without classical horizons.

Very compact objects with a hard and thermally emitting surface, such as gravastars, can be strongly constrained by observations [104, 105]. However, these constraints assume that the radiation channel is all in some Standard Model particles and is unlikely to strongly constrain boson stars, for example. 


\section{4. "No-hair" rises again}

Despite the several conceptual deaths of the no-hair hypothesis, there are good reasons to believe that astrophysical BHs are - to a good extent - described by the Kerr geometry:

- When a BH is surrounded by an accretion disk, the density of the disk is so small that the deviations from Kerr spacetime are tiny and, in many respects, can be neglected. Typically the disk can be understood as moving in a Kerr background geometry and used to infer properties of that background (as we explain in Section 5.4.3). More massive matter configurations may form (for instance, the dense disk discussed in [46]), but it is difficult to imagine that they persist for a significant fraction of the $\mathrm{BH}$ life.

- There is no indication that realistic collapse scenarios lead to the presence of substantial amounts of any of the hair discussed previously. Evolutions of the superradiant instability, for example (see Section 3.2 indicate that minimally coupled scalar clouds make up at most $\sim 30 \%$ of the system ADM mass, and that it is spread over a wide region 64] (therefore with negligible backreaction). Likewise, with the exception of boson stars and oscillatons [98, 106], none of the other ultracompact horizonless objects seem to arise as endpoints of gravitational collapse. Some of these objects (like wormholes, etc) are "cut and paste" or very contrived constructions, casting doubts on their ability to make actual predictions or doubts that their dynamics can ever resemble closely that of the objects they are supposed to mimick [107].

Note also that astrophysical BH-candidates come in all scales, from stellar mass to gigantic, supermassive objects. Boson stars and oscillatons, on the other hand, have a maximum mass (and compactness) dictated by the mass of some fundamental boson. It is thus hard to devise natural mechanisms to explain all observations.

- Even though hairy BH solutions or horizonless compact objects exist as equilibrium solutions of the field equations, some (many?) are dynamically unstable in a large portion of the parameter space: BHs in EYM theory are unstable [108]; BHs surrounded by minimally coupled massive scalars are suspected to be unstable in parts of the parameter space against an ergoregion instability [57, 109]; horizonless, spinning ultracompact object: * are unstable also against ergoregion instabilities [110 112]. In fact, some studies also suggest that any ultracompact object (spinning or not) is unstable [113, 114]

- It is possible that a carefully concocted horizonless object is stable on small timescales. However, for compactnesses extremely close to that of a $\mathrm{BH}$ (for example by modifying the geometry on Planck scales), the geometry is for many

* by which it is meant an horizonless object with a light ring.

$\#$ Curiously, all the stable configurations of a BH surrounded by a thin spherical shell of anisotropic fluid, described in 3.1 require that $R>3 M_{-}$, i.e., that the shell is outside the $\mathrm{BH}$ circular light ring. In this sense, it conforms to a "no short-hair" theorem [115], but can also be looked at as an example that ultracompact objects are generically unstable. 
purposes still that of a $\mathrm{BH}$ in GR. In fact, such objects might be very hard to distinguish, observationally, from BHs [11, 102, 116].

- In a large class of modified or extended theories of gravity, such as scalar-tensor or $f(R)$ theories, the Kerr geometry is still an equilibrium solution [117], albeit not necessarily unique. Insofar as tests of the background geometry are concerned, they are indistinguishable from GR BHs, but their fluctuations do also probe the underlying theory [118, making GW-based tests of the no-hair hypothesis relevant (see below for an expanded discussion on this point).

- Modified theories of gravity are typically parametrized by "small" coupling parameters, which induce parametrically small changes in the corresponding $\mathrm{BH}$ solutions. In addition, and perhaps even more relevant, ultracompact objects or Kerr BHs surrounded by small amounts of matter may be observationally indistinguishable from Kerr BHs: their ringdown waveform depends mostly on the properties of the unstable null circular geodesic, which is typically not significantly affected by "dirtiness" nor even by the presence or absence of an horizon [11, 116].

All the arguments above indicate that spinning BHs in our universe are likely to be described well by the Kerr geometry. The no-hair hypothesis is - and should be taken seriously In any case, observations hinting otherwise would provide clear signs of new physics or fields, and therefore measurements of "hair" are pursued with vigour.

\section{Tests of the "no-hair" hypothesis}

In order to test whether a compact object is described by the Kerr geometry, one needs to make observations. These can be, for instance, observations of stars orbiting around the compact dark companion, or observations of GWs from its oscillations. The former is an example of a non-dynamical test, which probes whether the stationary spacetime metric of the compact object is described by the Kerr solution. The latter, instead, is an example of a dynamical test, since it probes the compact object behaviour in a dynamical process.

\subsection{Multipole moments}

One of the most natural ways to test the spacetime metric of a compact object is to study - through astrophysical or GW observations - the motion of stellar objects in its surroundings. We here discuss the multipole expansions framework, which is probably the most appropriate to describe and perform these (non-dynamical) tests of the "no-hair" hypothesis. Multipole expansions have been first introduced in Newtonian mechanics, to describe the gravitational (or electrostatic) potential generated by a distribution of masses (or charges) in terms of a set of scalar quantities, the multipoles (see e.g., Ref. [119] and the first chapter of Ref. [120], and references therein). They have then been extended to GR [121-124]. 
Multipole expansions are a powerful tool to extract physical content from a (gravitational or electrostatic) potential, or from a spacetime metric. Indeed, the multipoles capture all the properties of the potential or of the metric, and in many cases the observable quantities can be expressed in terms of a multipolar decomposition. The measurement of the different multipole moments, through electromagnetic and GW observations, would allow the mapping of a $\mathrm{BH}$ spacetime.

In the following, we shall briefly introduce multipolar expansions in Newtonian gravity and in GR; further details are discussed in the Appendix.

5.1.1. Multipolar expansions in Newtonian gravity and in general relativity In Newtonian gravity, the gravitational potential $\Phi(t, \vec{x})$ in the exterior of a body with mass density $\rho(t, \vec{x})$ is the solution - in vacuum - of Poisson's equation $\nabla^{2} \Phi=4 \pi G \rho$. This solution can be written as a series expansion in $1 / r$ (where $r=|\vec{x}|)$, called multipolar expansion of the potential, which, in the case of a stationary, axisymmetric body, is

$$
\Phi(\vec{x})=-G \sum_{l=0}^{\infty} \frac{M_{l}}{r^{l+1}} P_{l}(\cos \theta),
$$

where $P_{l}(\cos \theta)$ are the usual Legendre polynomials, and

$$
M_{l}=\int \rho(\vec{x}) r^{l} P_{l}(\cos \theta) d^{3} x
$$

are the multipole moments of the body. The first moments are well known: $M_{0}=M$ is the body's mass; $M_{1}$ is its dipole moment, vanishing in the center-of-mass frame; $M_{2}=Q$ is the quadrupole moment, $M_{3}$ is the octupole moment, and so on. Introducing dimensionless multipole moments $J_{l}=-M_{l} /\left(M R^{l}\right)$, where $R$ is a characteristic length of the body (in the case of the Earth, $R$ is the equatorial radius),

$$
\Phi=-G\left[\frac{M}{r}-J_{2} \frac{M R^{2}}{r^{3}} P_{2}(\cos \theta)+\ldots\right] .
$$

The multipole moments of an astronomical body are a measure of its departure from spherical symmetry. Generally, planets and stars rotate so slowly that their deviation from spherical symmetry is tiny (other sources of asymmetry are even smaller). For instance, for the Earth $J_{2}=1.083 \times 10^{-3}$, and the $l>2$ dimensionless multipoles are at least a thousand times smaller. These quantities have been determined by studying the motion of satellites orbiting around the Earth [125-127]. A depiction of the gravitational field of the Earth as reconstructed with data from these mission is shown in Fig. 5, where differences in the gravitational field across the globe are represented by elevation and color.

The generalization of multipolar expansions to GR is not straightforward, due to the non-linearity of Einstein's equations. The relativistic multipole moments of a stationary, asymptotically flat spacetime have been defined by Geroch and Hansen [121, 122], with a complex mathematical construction that allows to describe the deviation of the asymptotic geometry from flatness in terms of two sets of tensorial quantities evaluated at the point at infinity: the mass multipole moments and the current multipole moments 


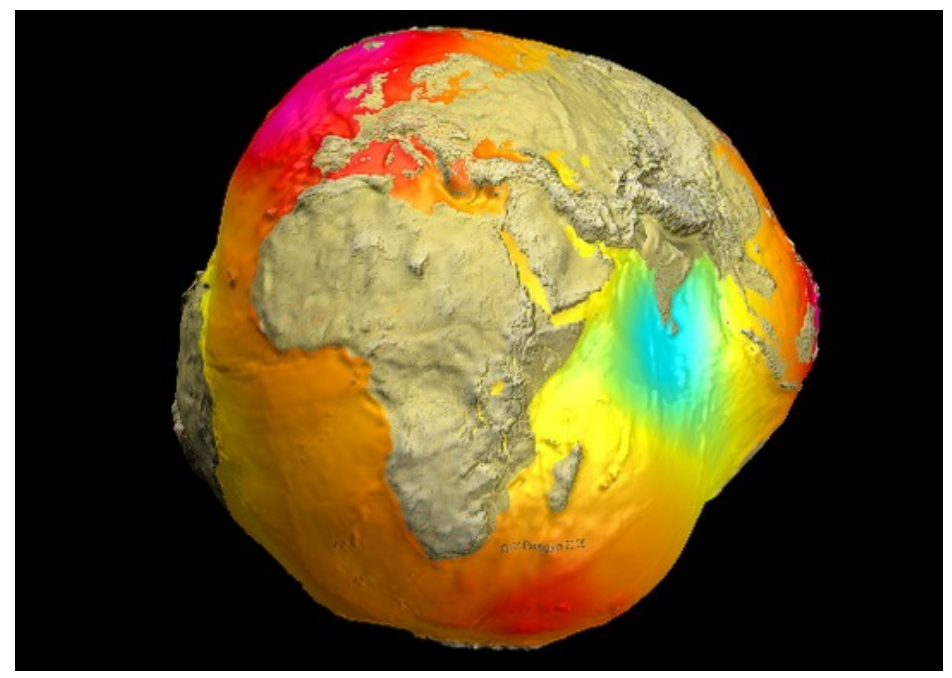

Figure 5. The gravitational field of the Earth (known as the Potsdam Potato), based on data from the LAGEOS, GRACE, and GOCE satellites and surface data. Gravitational field strength is represented by elevation and color. Credit: CHAMP [128, GRACE [129, Research Center for Geophysics (GFZ) [130, NASA [131, DLR [132].

(see the Appendix). In the Newtonian limit, the mass multipole moments reduce to the moments in Newtonian theory $\dagger$. It has been shown that the spacetime is uniquely determined by its multipole moments [133 135]: in other words, they completely characterize the spacetime geometry outside any stationary body. It has also been shown (in the axisymmetric case) that it is possible to reconstruct the full spacetime from any "well-behaved" set of relativistic multipole moments [136, 137].

As in the Newtonian case, when the body (and the spacetime) is axisymmetric, the relativistic (mass and current) multipoles reduce to a set of scalar quantities $\left(M_{l}, J_{l}\right)$ [122, 124]; if the spacetime is reflection-symmetric (i.e., symmetric with respect to a reflection on the equatorial plane), the odd mass moments and the even current moments identically vanish: $M_{2 l+1}=S_{2 l}=0$. As in the Newtonian case, $M_{0}=M$ is the mass, $M_{2}=Q$ is the quadrupole moment; moreover, $S_{1}=J$ is the angular momentum. For the Kerr spacetime,

$$
\begin{aligned}
& M_{2 l}=(-1)^{l} M a^{2 l} \\
& S_{2 l+1}=(-1)^{l} M a^{2 l+1} .
\end{aligned}
$$

Notice that this expression is a manifestation of ("no-hair") Theorem 1 (Sec. 1.2), as it fixes all multipole moments as function of two parameters only.

An alternative definition of relativistic multipole moments of stationary, asymptotically flat spacetimes has been introduced by Thorne [123]. This is an extension of the standard procedure of extracting the mass and the angular momentum from the far-field limit of the spacetime metric [138]. In Thorne's construction, all multipole

$\dagger$ The current multipole moments do not appear in Newtonian theory, because they do not affect the motion of masses. 
moments can be read out from the asymptotic spacetime metric. In the axisymmetric case

$$
\begin{aligned}
g_{00} & =-1+\frac{2 M}{r}+\sum_{l \geq 2} \frac{2}{r^{l+1}}\left[M_{l} P_{l}(\cos \theta)+\left(l^{\prime}<l \text { harmonics }\right)\right] \\
& =-1+\frac{2 M}{r}+\frac{2 Q}{r^{3}} P_{2}(\cos \theta)+\ldots \\
g_{0 \phi} & =-2 \sin ^{2} \theta \sum_{l \geq 1} \frac{1}{r^{l}} \frac{S_{l}}{l}\left[P_{l}^{\prime}(\cos \theta)+\left(l^{\prime}<l \text { harmonics }\right)\right] \\
& =-\sin ^{2} \theta\left(\frac{2 J}{r}+\frac{2 S_{3}}{3 r^{3}} P_{3}^{\prime}(\cos \theta)+\ldots\right) .
\end{aligned}
$$

This definition requires that the coordinate system belongs to a special class, the "asymptotically Cartesian and mass centered" (ACMC) coordinates, which means that it becomes Minkowski with sufficient rapidity at large radii (see the Appendix for details), and that the origin of the space coordinates lies at the center of mass of the source. As long as the coordinates are ACMC, the multipole moments $\left(M_{l}, S_{l}\right)$ are coordinate-independent. Moreover, it has been shown that the definitions of relativistic multipole moments given by Geroch-Hansen and by Thorne are actually coincident 139] 舟

In the case of a weak-field source, the multipole moments of a stationary spacetime can also be expressed as integrals over the source of the energy and momentum densities [140], i.e. (in the axisymmetric case) by Eq. (5.2) for mass multipoles, and a similar expression for current multipoles [141, 142]. This definition can be extended, using properly defined "effective" (energy and momentum) densities, to the case of strong-field sources, as long as the source can be covered by a so-called "de Donder" coordinate frame [123]; this is the case, for instance, of compact stars. Multipole moments of BHs, instead, can only be defined in terms of their asymptotic geometry.

Given a spacetime metric expressed in a specific coordinate frame, there is a simple method to compute the Geroch-Hansen multipole moments of the spacetime. This procedure is based on Ryan's formula [143], which was introduced as a phenomenological tool to express observable quantites in terms of multipole moments (and will be discussed in more detail in Sec. 5.3), but is also a powerful computational tool. One computes the energy of (geodesic) circular orbits as a function of the orbital frequency $E(\nu)$. A particle moving in that geodesic would emit GWs at frequency $f=2 \nu$, and the so-called "gravitational spectrum", i.e. the amount of GW energy emitted per logarithmic interval of frequency, is $\Delta E(f)=f d E_{g w} / d f=-\nu d E / d \nu$. Ryan has found a general, explicit expression of $\Delta E(f)$ in terms of the multipole moments of the spacetime (Eq. 5.19) in Sec. 5.3); comparing the function $\Delta E(f)$ with this expression, one can extract the entire set of (Geroch-Hansen) multipole moments. This approach can be useful to express the

$\ddagger$ Note that Thorne [123] uses a normalization for multipole moments different than that of Geroch and Hansen [121, 122. In Eqns. 5.5 we follow the conventions of Geroch-Hansen, which is also adopted in most of the recent literature on the subject. 
asymptotic expansion of the metric - which in general in not expressed in an ACMC coordinate frame - in terms of the gauge-invariant multipole moments, for instance when the metric is the result of a numerical integration [144 147], or of a perturbative computation [148, 149]. When the metric is known in ACMC coordinates, instead, the multipoles can be extracted by comparison with Thorne's expansion (5.5), as e.g. in [150].

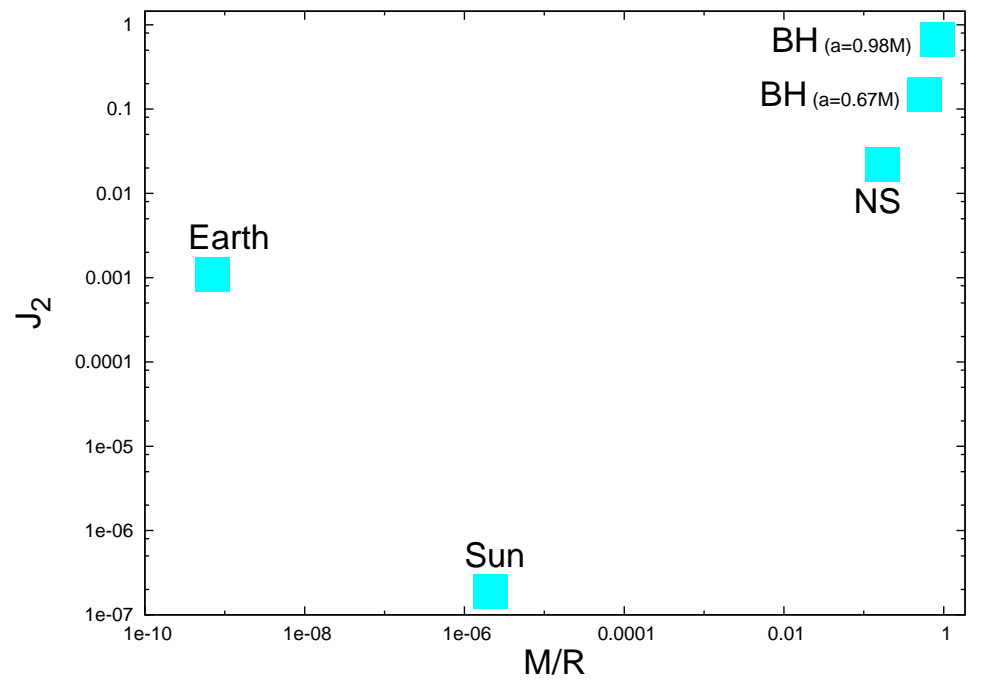

Figure 6. Typical values of the normalized quadrupole moments for the Earth, the Sun, a neutron star and two examples of BH. The neutron star [151, 152] has a mass $M=1.4 M_{\odot}$, a radius $R=12 \mathrm{~km}$, is spinning at $716 \mathrm{~Hz}$ (the maximum observed spin rate [153]) and is described using the equation of state of Ref. 154. The BHs are the final BH in the GW150914 coalescence [1, having $a=0.67 M$, and a near-extremal BH with $a=0.98 M$.

5.1.2. The multipole moments of some astrophysical objects To conclude this discussion, we show in Figure 6 typical values of the normalized quadrupole moment $J_{2}=-Q /\left(M R^{2}\right)$, as a function of the compactness $M / R$, for different astrophysical objects: the Earth and the Sun [155]; a neutron star; the final BH observed in the first LIGO detection [1], having $a=0.67 M$ and $R=1.74 M$; a near-extremal BH with $a=0.98 M$ (and thus $R=1.20 M$ ). This figure shows that the normalized quadrupole moment of astrophysical objects comes in a variety of ranges, and that therefore the $\mathrm{BH}$ multipole moments are not accidentally shared by many other objects.

However, we should also add that (exotic) ultra-compact objects can have multipole moments arbitrarily close to that of BHs. For example, in the ultra-compact limit, the mass quadrupole moment of gravastars is [156]

$$
Q=\frac{J^{2}}{M}\left(1+\frac{8}{45 \log (1-2 M / R)}\right),
$$




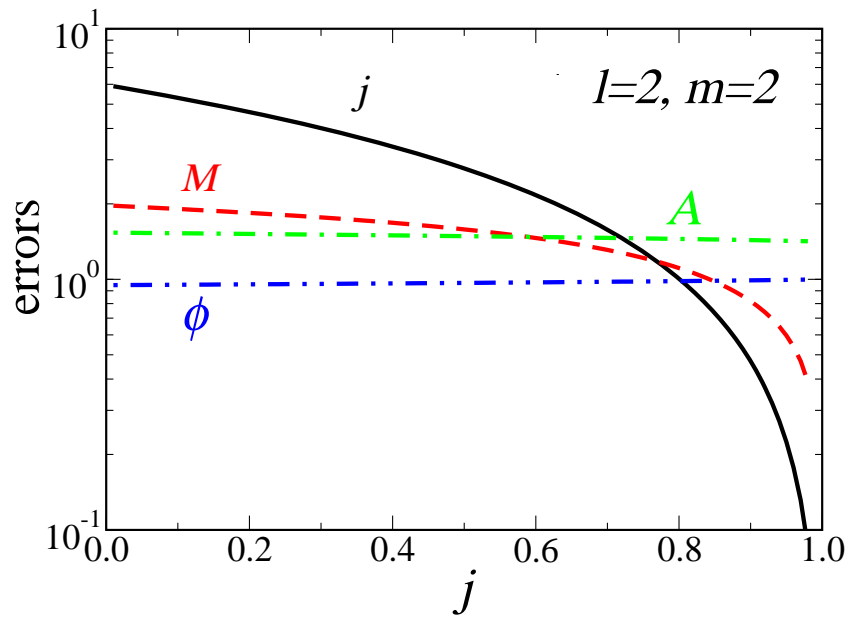

Figure 7. Errors (multiplied by the signal-to-noise ratio $\rho$ ) in measurements of different parameters for the fundamental $l=m=2$ mode as functions of the angular momentum parameter $j$. Solid (black) lines give $\rho \sigma_{j}$, dashed (red) lines $\rho \sigma_{M} / M$, dotdashed (green) lines $\rho \sigma_{A} / A$, dot-dot-dashed (blue) lines $\rho \sigma_{\phi}$, where $\sigma_{k}$ denotes the estimated rms error for variable $k, M$ denotes the mass of the $\mathrm{BH}$, and $A$ and $\phi$ denote the amplitude and phase of the wave. From Ref. [38].

showing that it can be arbitrarily close to that of a $\mathrm{BH}$ with the same mass, i.e., $Q=J^{2} / M$. This somewhat contrived example shows that the law (5.4) is not, by itself, evidence for the existence of a horizon, but the alternatives usually invoke somewhat more exotic physics.

\subsection{Dynamical tests with gravitational waves: ringdown}

5.2.1. Using two or more modes to test the Kerr hypothesis Tests of the no-hair hypothesis based on the motion and observation of stars at large distances probe the background geometry in the weak-field regime. A multipolar decomposition is meaningful here, because the contribution of higher multipoles to the motion of stars is suppressed. Nevertheless, such tests are unable to probe different theories with the same background solution. To probe the dynamical content of the field equations, dynamical tests are necessary, and GWs are the ideal tool for this.

As we discussed in Section 2, the vibration modes of BHs in GR are completely determined by the two parameters specifying the Kerr solutions. In any other theory with the same Kerr background, the functional relation among modes would be different. Thus, conceptually one can proceed as follows: measure the dominant mode of oscillation, which is characterized by a ringing frequency $\omega$ and a damping time $\tau$. In GR, the dominant mode is invariably (when the oscillations are the result of a $\mathrm{BH}$ binary coalescence, which is likely to be the most efficient way to excite the QNMs) that with $l=m=2, n=0$ mode, of frequency $\omega_{220}$ and damping time $\tau_{220}$. In the 
Schwarzschild limit these are the numbers shown in equation (2.3). These numbers allow one to determine the (redshifted) mass and angular momentum of the BH. Now measure the most important subdominant mode (typically $l=m=3, n=0$ ) and check that it is located at the GR prediction. One thus has at hand a null test of GR based on the observation of ringdown modes of BHs.

In practice, there are measurement errors associated with the different sources of noise in the detector, and each of the stages in the test carries associated uncertainties. These uncertainties are best described in units of the "signal-to-noise" ratio (SNR) $\rho$ [157, 158]. As a rule-of-thumb, a total $\rho \gtrsim 8$ is required for a detection. In the following we focus on the SNR for the ringdown part of the signal only. Fortunately, accurate measurements of the mass and angular momentum of BHs are feasible [38, 159]: for detection of the fundamental $l=m=2$ bar mode, for example, Figure 7 shows the estimated error (multiplied by $\rho$ ) in measuring the mass $M$, angular momentum parameter $j \equiv a / M$, QNM amplitude $A_{l m n}$, and phase $\phi_{l m n}$ (see equation (2.1) for definitions of these quantities and Ref. [38] for further details). At large $\rho$, the uncertainties are well approximated by [38, 160]

$$
\sigma_{j}=\frac{2}{\rho}\left|\frac{Q_{l m n}}{Q_{l m n}^{\prime}}\right|, \quad \sigma_{M}=\frac{2}{\rho}\left|\frac{M Q_{l m n} f_{l m n}^{\prime}}{f_{l m n} Q_{l m n}^{\prime}}\right|,
$$

where primes stand for derivatives with respect to the dimensionless angular momentum $j$, and can be performed with the help of the fits in Eqs.(2.4)-(2.5), or taken from the numerical data [2, 37, 38]. Even for marginal detection events, the accuracy of mass and spin measurements is at the level of $10 \%$ or better.

For large signal-to-noise ratios, one enters the regime where a second mode can be disentangled in the signal. To understand what criteria needs to be met, consider first disentangling frequencies. The error associated with the direct measurement of the frequency and damping time of one mode (" 1 ") in the signal is [38, 160]

$$
\begin{aligned}
\rho \sigma_{f_{1}} & =\frac{1}{2 \sqrt{2}}\left\{\frac{f_{1}^{3}\left(3+16 Q_{1}^{4}\right)}{\mathcal{A}_{1}^{2} Q_{1}^{7}}\left[\frac{\mathcal{A}_{1}^{2} Q_{1}^{3}}{f_{1}\left(1+4 Q_{1}^{2}\right)}+\frac{\mathcal{A}_{2}^{2} Q_{2}^{3}}{f_{2}\left(1+4 Q_{2}^{2}\right)}\right]\right\}^{1 / 2}, \\
\rho \sigma_{\tau_{1}} & =\frac{2}{\pi}\left\{\frac{\left(3+4 Q_{1}^{2}\right)}{\mathcal{A}_{1}^{2} f_{1} Q_{1}}\left[\frac{\mathcal{A}_{1}^{2} Q_{1}^{3}}{f_{1}\left(1+4 Q_{1}^{2}\right)}+\frac{\mathcal{A}_{2}^{2} Q_{2}^{3}}{f_{2}\left(1+4 Q_{2}^{2}\right)}\right]\right\}^{1 / 2} .
\end{aligned}
$$

These errors refer to mode " 1 " in a pair. By considering the "symmetric" case $\phi_{1}=\phi_{2}=0$, the errors on $f_{2}$ and $\tau_{2}$ are simply obtained by exchanging indices $(1 \leftrightarrow 2)$.

A natural criterion (á la Rayleigh) to resolve frequencies and damping times is

$$
\left|f_{1}-f_{2}\right|>\max \left(\sigma_{f_{1}}, \sigma_{f_{2}}\right), \quad\left|\tau_{1}-\tau_{2}\right|>\max \left(\sigma_{\tau_{1}}, \sigma_{\tau_{2}}\right) .
$$

In interferometry this would mean that two objects are (barely) resolvable if "the maximum of the diffraction pattern of object 1 is located at the minimum of the diffraction pattern of object 2". We can introduce two "critical" SNRs required to resolve frequencies and damping times,

$$
\rho_{\text {crit }}^{f}=\frac{\max \left(\rho \sigma_{f_{1}}, \rho \sigma_{f_{2}}\right)}{\left|f_{1}-f_{2}\right|}, \quad \rho_{\text {crit }}^{\tau}=\frac{\max \left(\rho \sigma_{\tau_{1}}, \rho \sigma_{\tau_{2}}\right)}{\left|\tau_{1}-\tau_{2}\right|},
$$




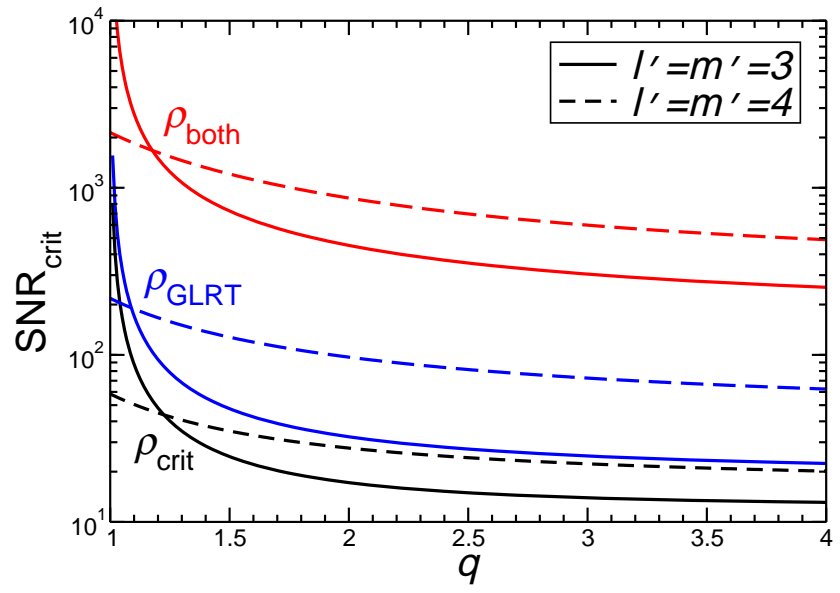

Figure 8. Minimum SNR (in ringdown only) required to resolve two modes, as function of the binary's mass ratio $q$. If $\rho>\rho_{\text {GLRT }}$ we can tell the presence of a second mode in the waveform, if $\rho>\rho_{\text {crit }}$ we can resolve either the frequency or the damping time, and if $\rho>\rho_{\text {both }}$ we can resolve both. Mode " 1 " is assumed to be the fundamental mode with $l=m=2$; mode " 2 " is either the fundamental mode with $l=m=3$ (solid lines) or the fundamental mode with $l=m=4$ (dashed lines). From Ref. [160].

and recast our resolvability conditions as

$$
\rho>\rho_{\text {crit }}=\min \left(\rho_{\text {crit }}^{f}, \rho_{\text {crit }}^{\tau}\right), \quad \rho>\rho_{\text {both }}=\max \left(\rho_{\text {crit }}^{f}, \rho_{\text {crit }}^{\tau}\right) .
$$

The first condition implies resolvability of either the frequency or the damping time, the second implies resolvability of both.

A related question is the magnitude of the signal-to-noise ratio in order to detect a multi-mode signal, and to resolve two signals of different amplitudes. This problem was studied in Ref. [160], and quantified by deriving a critical signal-to-noise ratio for amplitude resolvability $\rho_{\text {GLRT }}$ based on the generalized likelihood ratio test. This criteria is almost (but not quite) equivalent to requiring that the second, sub-dominant mode alone has enough energy that it could be detected on its own.

The different signal-to-noise ratios required to resolve the two modes of a final $\mathrm{BH}$ which is the end-product of an unequal-mass merger of two BHs, is shown in Fig. 8 . The figure shows that tests of the no-hair hypothesis require large, but not-too-largeto-be-impossible signal-to-noise ratios.

The implementation of this method in an actual search pipeline for two planned GW detectors ET and NGO was described in Ref. [161]. The authors consider a theoryindependent parametrization of the QNM frequencies given by

$$
\begin{aligned}
& \omega_{l m}=\omega_{l m}^{(\mathrm{GR})}\left(1+\Delta \hat{\omega}_{l m}\right) \\
& \tau_{l m}=\tau_{l m}^{(\mathrm{GR})}\left(1+\Delta \hat{\tau}_{l m}\right),
\end{aligned}
$$

where $\omega_{l m}^{(\mathrm{GR})}$ are the values of the fundamental QNM frequencies and damping times of 

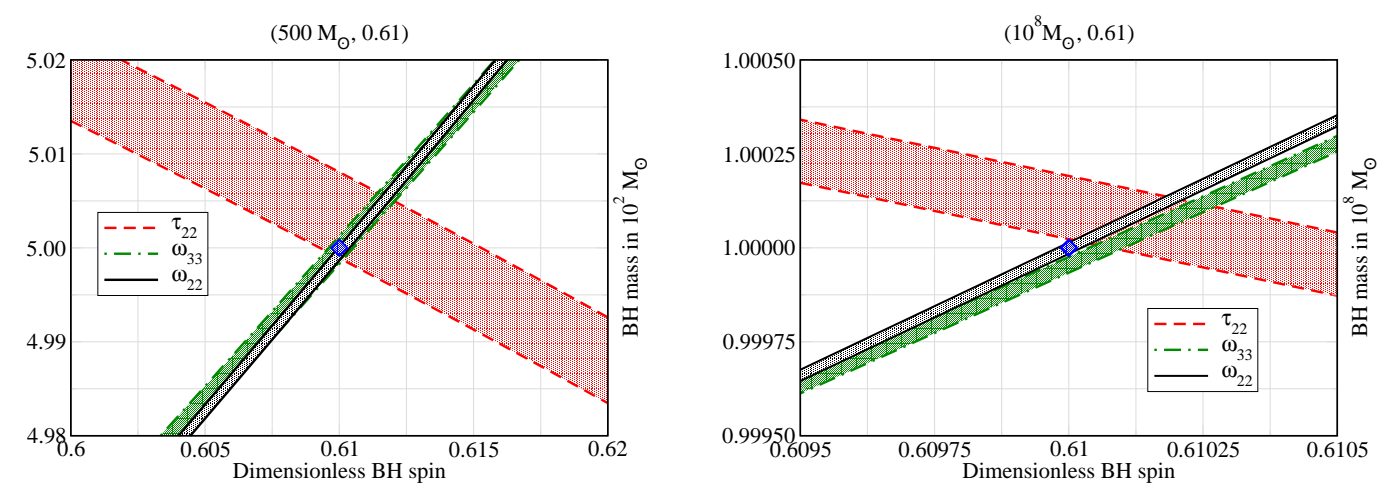

Figure 9. Projections in the $(M, j)$-plane of the $90 \%$ confidence limits on $\omega_{22}, \tau_{22}$ and $\omega_{33}$ (blue, blue dotted and red lines respectively) for injections of signals consistent with GR for $M=500 M_{\odot}$ (left at $125 \mathrm{Mpc} ; \mathrm{SNR}=2888$ ), and $M=10^{8} M_{\odot}$ (right at $1 \mathrm{Gpc}$; SNR $=115154$ ). The injected value is denoted in each case by a diamond. Taken from Ref. [161].

Kerr BHs, and $\Delta \hat{\omega}_{l m}, \Delta \hat{\tau}_{l m}$ parametrize the relative deviations to these numbers. The $\mathrm{BH}$ is assumed to be the endpoint of a mass ratio $q=2,10$ binary $\mathrm{BH}$ merger and its spin is assumed to be $j=0.6,0.26$.

When the signal is described by GR, estimates of mass and spin from, say, three modes will be consistent and yield (generically accurate) measurements of these quantities. This is depicted in Fig. 9, the three estimates intersect at a common point, which will be the best estimate for the mass and spin of the final BH (the injected signal corresponds to a $\mathrm{BH}$ of mass $\left(500,10^{8}\right) M_{\odot}$ for $\mathrm{NGO}$ and ET respectively, and the best estimate is very close to the injected value).
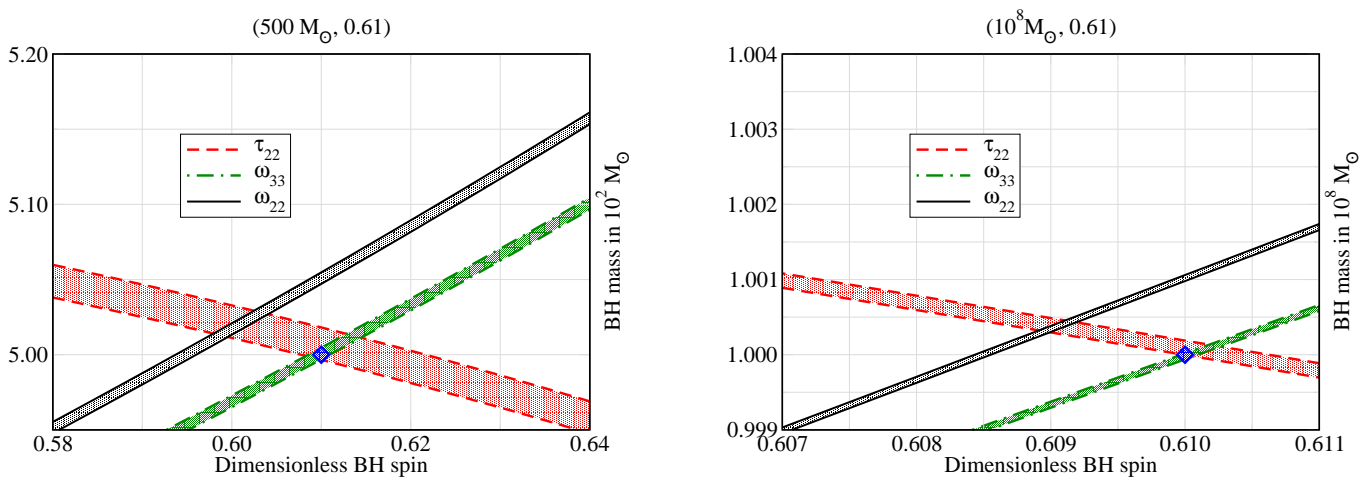

Figure 10. Projections in the $(M, j)$-plane of the $90 \%$ confidence limits on $\omega_{22}$, $\tau_{22}$ and $\omega_{33}$ (blue, blue dotted and red lines, respectively) for non-GR injections of $M=500 M_{\odot}$ (left at $125 \mathrm{Mpc}$ for the ET telescope; with $\Delta \hat{\omega}_{22}=-0.01, \mathrm{SNR}=2867$ ) and and $M=10^{8} M_{\odot}$ (right at $1 \mathrm{Gpc}$ for NGO telescope; with $\Delta \hat{\omega}_{22}=-0.001$, SNR $=115130)$. The injected value is denoted in each case by a diamond. Taken from Ref. [161. 

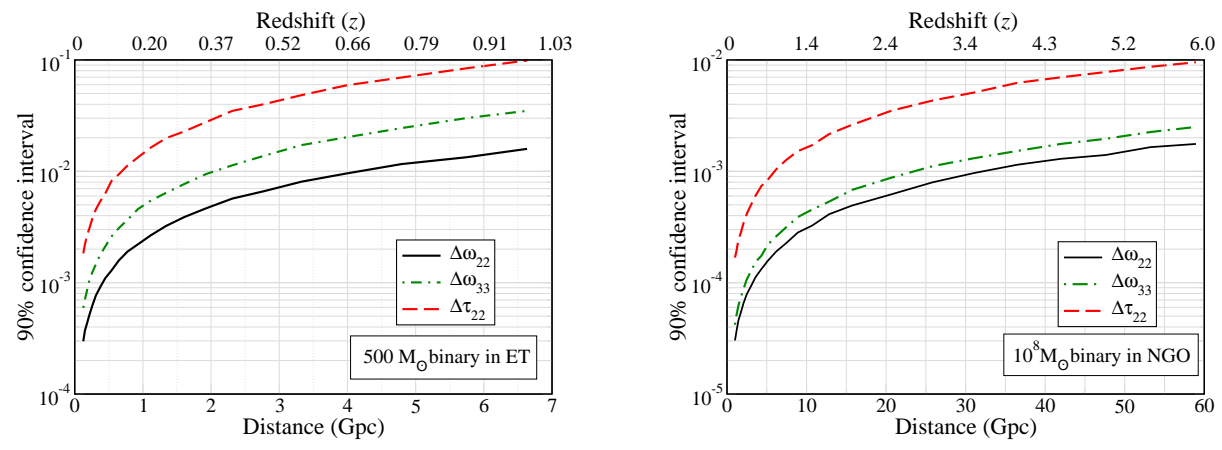

Figure 11. Width of the $90 \%$ confidence intervals for $\Delta \hat{\omega}_{22}, \Delta \hat{\omega}_{33}$ and $\Delta \hat{\tau}_{22}$ (blue, red and blue dotted lines respectively) against luminosity distance for injections of 500 (left, ET) and $10^{8} M_{\odot}$ (right, NGO). Taken from Ref. [161.

By contrast, if the signal is inconsistent with GR the intersections of the confidence regions will not agree, as in Fig. 10 .

The accuracy with which the deviation constants can be estimated and the mode parameters resolvable is shown in Figure 11. For each BH system considered, the width of the $90 \%$ confidence intervals for the extracted values of $\Delta \hat{\omega}_{l m}$ and $\Delta \hat{\tau}_{l m}$ were plotted against luminosity distance, for injections with GR waveforms.
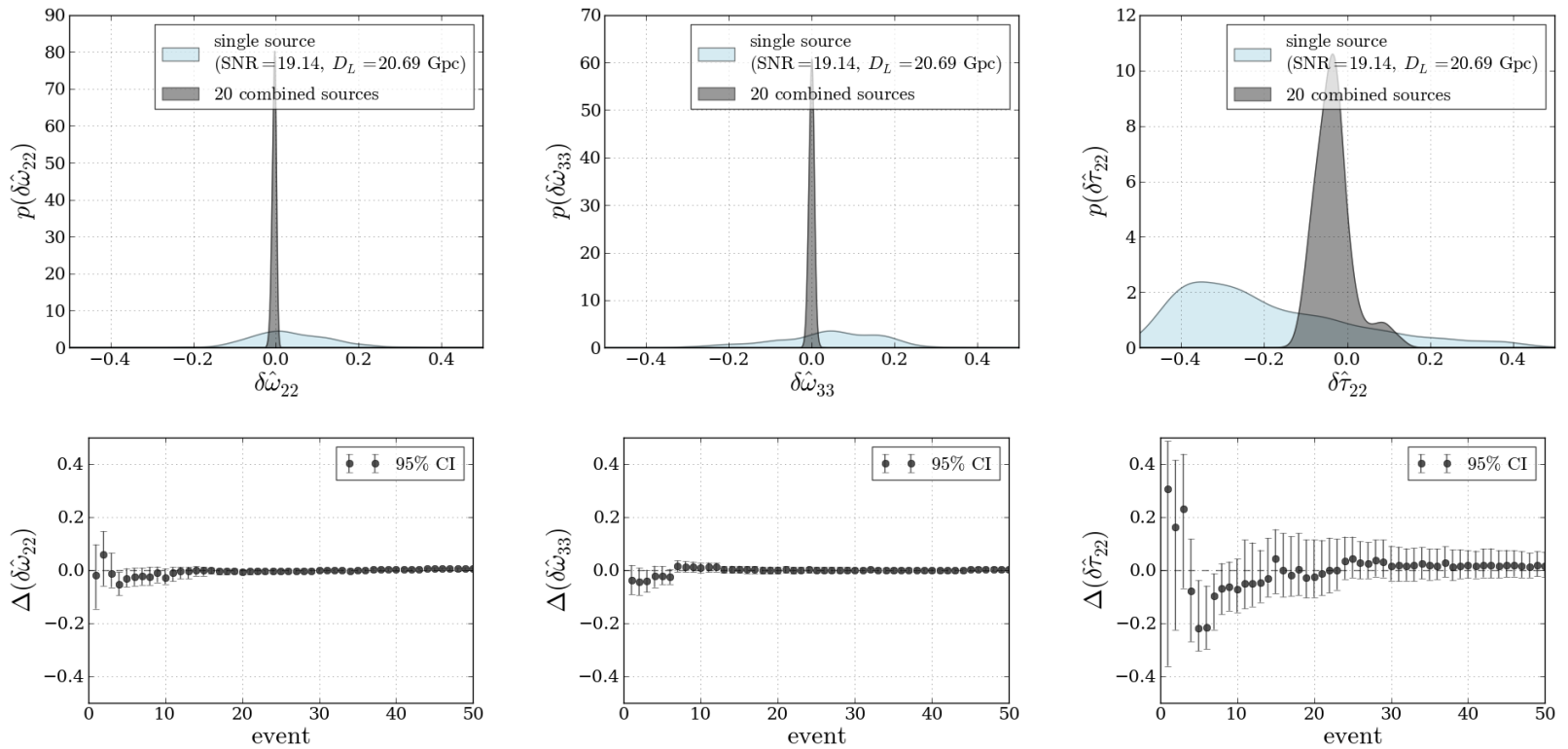

Figure 12. Top panels: Posterior density functions for $\delta \hat{\omega}_{22}$ (left), $\delta \hat{\omega}_{33}$ (middle), and $\delta \hat{\tau}_{22}$ (right), both for a single source at a distance of $20.69 \mathrm{Gpc}(z=2.47)$ with an SNR of 19.14, and for a catalog of 20 sources. Bottom: Evolution of medians and 95\% confidence intervals of PDFs as more and more sources are included. Taken from Ref. [162].

The previous results all assume comparatively large signal-to-noise ratios. This analysis was recently extended to multiple detections with larger noise, using a model 
selection scheme called TIGER (Test Infrastructure for GEneral Relativity), for the ET telescope [162].

As an example, assume that GR is correct $\left(\delta \hat{\omega}_{22}=\delta \hat{\omega}_{33}=\delta \hat{\tau}_{22}=0\right)$. How would GW observations of ringdown actually constrain the deviation parameters? Results are summarized in Fig. 12. The top panels show the probability distribution function (PDF) both for an example single source at $D_{\mathrm{L}}=20.69 \mathrm{Gpc}(z=2.47)$, and for a catalog of 20 sources. As expected, the single-source PDFs are quite wide and uninformative. For $\delta \hat{\omega}_{22}$ and $\delta \hat{\omega}_{33}$, with 20 sources the PDFs become strongly peaked, with very little bias. There is a clear advantage in using all available detections.

If the $\mathrm{BH}$ progenitor is known with some accuracy, further tests are possible, even with a single ringdown mode: the inspiral phase allows, in principle, the mass and spin of the two components to be determined. GR then predicts a well-defined final BH with a certain mass and spin, a prediction which can be tested using the dominant ringdown mode [163]. In fact, in some cases it might even be possible to infer the properties of the progenitor from ringdown observations [164, 165].

These results were recently used, in conjunction with population synthesis models of the formation and evolution of BH binaries, to estimate how many detections would yield tests of GR. The results are optimistic for space-based detectors or future generations of Earth-based ones [166].

5.2.2. Constraining alternative theories Up to now, we studied how two modes can test GR. We can turn this around and use measurements of two (or more) ringdown modes to constraint specific modified theories, converting the errors (5.8)-(5.9) on the frequency and damping time to errors on physical quantities by using a simple propagation of errors [12]. Specifically, let $M, j=a / M$ be the mass and dimensionless angular momentum of the $\mathrm{BH}$, and $Q$ an extra parameter which presumably enters the description of the geometry. Since $Q$ measures deviations from GR or from the Kerr geometry, we will always take it to be small. Then

$$
\sigma_{X}=\frac{\partial X}{\partial M} \sigma_{M}+\frac{\partial X}{\partial \chi} \sigma_{\chi}+\frac{\partial X}{\partial Q} \sigma_{Q}
$$

where $X=\left(f_{1}, f_{2}, \tau_{1}\right)$. It is straightforward to solve the system of three equations above for $\sigma_{M}, \sigma_{\chi}$ and $\sigma_{Q}$; this yields

$$
\begin{aligned}
& \rho \sigma_{M}=F_{1}\left(f_{1}, f_{2}, \tau_{1}, \mathcal{A}_{2} / \mathcal{A}_{1}\right), \\
& \rho \sigma_{\chi}=F_{2}\left(f_{1}, f_{2}, \tau_{1}, \mathcal{A}_{2} / \mathcal{A}_{1}\right), \\
& \rho \sigma_{Q}=F_{3}\left(f_{1}, f_{2}, \tau_{1}, \mathcal{A}_{2} / \mathcal{A}_{1}\right),
\end{aligned}
$$

where $F_{i}$ are, usually, cumbersome analytical functions. Finally, and because $Q$ is small, we can view $\sigma_{Q}$ as an upper bound on the quantity $Q$ itself and use it to estimate the constraint that can be imposed by a ringdown detection with a certain SNR $\rho$. This strategy was used to study constraints on the electric charge of BHs, resulting in the constraint $\frac{|Q|}{M} \lesssim 0.1 \sqrt{\frac{100}{\rho}}[12]$. A similar procedure can used to constraint the magnitude 
of the Gauss-Bonnet coupling constant, or of any other theories, as long as the QNMs are well understood in these theories [167.

5.2.3. Environmental effects The QNMs of BHs are intimately connected to the null circular geodesic, or light ring [11, 36, 116, 168]. In addition, orbits that pass inside the light ring must plunge into the $\mathrm{BH}$. Indeed, the innermost stable circular orbit (ISCO) for example, is always outside the light ring. As such, stars or matter debris composing accretion disks are not expected to populate the region close to the light ring. In turn, this means that "environmental effects" (i.e., the impact of matter surrounding the BH) are expected to have a small impact on the QNMs of BHs and consequently on tests of the Kerr-hypothesis.

An exhaustive list of environmental effects, such as the impact of electric charge, accretion disks, dark matter, cosmological constant, etc are presented and quantified in Refs. 11, 168. The results are summarized in Table 1. These effects represent the ultimate precision with which one can test the no-hair hypothesis, in the absence of more detailed knowledge about the matter surrounding the BH. To summarize, no-hair tests are possible with ringdown waves from perturbed BHs, with threshold signal-to-noise ratios shown in Fig. 8. Third-generation detectors will be able to perform accurate tests of GR, or possibly infer details of the environment surrounding BHs.

5.2.4. QNMs as a probe of the event horizon As we remarked, the $\mathrm{BH}$ ringdown is a dynamical probe of the underlying theory, and is tightly connected to the light ring properties. It is not surprising therefore that ringdown probes the region very close to the event horizon [169 171]. However, precisely because ringdown is related to the light ring, ultra-compact objects with light rings may also display similar signals [11, 116].

Table 1. Upper limits on the environmental corrections to the BH QNMs. We define $\delta_{R, I}=1-\omega_{R, I} / \omega_{R, I}^{(0)}$, where $\omega_{R, I}$ is the real (imaginary) part of the ringdown frequency in the presence of environmental effects, whereas $\omega_{R, I}^{(0)}$ is the same for an isolated $\mathrm{BH}$ with the same total mass. Conservative environmental reference values are $q=10^{-3}, B=10^{8}$ Gauss, $\rho_{3}^{\mathrm{DM}}=\rho_{\mathrm{DM}} /\left(10^{3} M_{\odot} / \mathrm{pc}^{3}\right)$. We assume a ShakuraSunyaev disk model with viscosity parameter $\alpha=0.1$ and Eddington ratio $f_{\mathrm{Edd}}=10^{-4}$ $\left(f_{\mathrm{Edd}}=1\right)$ for thick and thin disks, respectively. The spherical and ring-like matter distributions have mass $\delta M \sim 10^{-3} M$. The scaling with the parameters is shown in Ref. [11. Taken from Ref. [168.

\begin{tabular}{c|cc}
\hline Correction & $\left|\delta_{R}\right|[\%]$ & $\left|\delta_{I}\right|[\%]$ \\
\hline spherical near-horizon distribution & 0.05 & 0.03 \\
ring at ISCO & 0.01 & 0.01 \\
electric charge & $10^{-5}$ & $10^{-6}$ \\
magnetic field & $10^{-8}$ & $10^{-7}$ \\
gas accretion & $10^{-11}$ & $10^{-11}$ \\
DM halos & $10^{-21} \rho_{3}^{\text {DM }}$ & $10^{-21} \rho_{3}^{\text {DM }}$ \\
cosmological effects & $10^{-32}$ & $10^{-32}$ \\
\hline
\end{tabular}




\subsection{Dynamical tests with gravitational waves: inspiral}

As shown by Ryan [143, the multipole moments of a central object which is assumed to be stationary, axisymmetric and reflection-symmetric, can be measured by looking at the motion of a test body orbiting around the central object. Indeed, observable, gaugeinvariant quantities can be expressed in terms of the multipole moments, as defined by Geroch and Hansen. These quantities are the GW spectrum, i.e. (as mentioned in Sec. 5.1.1) the amount of GW energy emitted per logarithmic interval of frequency; the perihastron precession and the orbital plane precession ("epyciclic frequencies"); the number of cycles of the GW signal emitted per logarithmic interval. For instance, the GW spectrum is

$$
\begin{aligned}
\Delta E(f) & =f \frac{d E_{g w}}{d f} \\
& =\mu\left(\frac{1}{3} v^{2}-\frac{1}{2} v^{4}+\frac{20}{9} \frac{S_{1}}{M^{2}} v^{5}+\left(-\frac{27}{8}+\frac{M_{2}}{M^{3}}\right) v^{6}+\ldots\right)
\end{aligned}
$$

where $f$ is the frequency of the emitted GWs (twice the orbital frequency $\nu$ ), $\mu$ is the mass of the test body, and $v=(\pi M f)^{1 / 3}$. Since the frequency is associated with the Killing vector of the stationary spacetime, all of these quantities are gauge-invariant. Note that the post-Newtonian expansion (5.19) is only accurate for $v \ll 1$; near the ISCO, $v \sim(6)^{-1 / 2}$ (or larger for rotating BHs) and higher-order terms should be included in the expansion [172].

Ryan's construction [143], in principle, seems to be an extremely powerful phenomenological tool. GW and electromagnetic observations can be used to measure the multipole moments of the central object, thus providing a mapping of the spacetime. In particular, the moments can be extracted from the GW signal emitted by an extreme mass-ratio inspiral (EMRI), i.e., a stellar-mass BH inspiralling around a supermassive $\mathrm{BH}$ - a target source for space-based detectors such as eLISA [173, 174]. A comparison with the moments of Kerr's solution (5.4) would provide a test of the "no-hair" hypothesis.

In practice, the ambitious program of "mapping the $\mathrm{BH}$ spacetime" with its multipole moments turned out to be less practical than expected. Firstly, it has been observed that multipole expansions are poorly convergent in the strong-field region near the $\mathrm{BH}$ horizon, where a large number of terms is required to describe the spacetime [175. Although the detection of an EMRI waveform by an eLISA-like detector could allow to measure the quadrupole $Q=M_{2}$ with good accuracy [176, 177], a deviation from Kerr spacetime can affect the higher-order multipoles as well, which are difficult to measure independently. Secondly, the beautiful expression of $\Delta E$ in terms of the multipole moments $(5.19)$ is useless if we do not also know the geodesic motion and the emitted GW flux, which are required to compute the gravitational waveform [178]; and both geodesic motion and GW flux are very difficult to describe for a non-Kerr spacetime with arbitrary multipole moments. We also remark that the GW flux depends on the dynamical equations of gravity. 
Several different approaches have been developed to deal with these concerns. Ryan's construction has been extended to specific modified gravity theories, such as scalar-tensor gravity [179] and, to some extent, to EDGB gravity [180], in order to make the "theory bias" towards general relativity less severe. Other authors have constructed parametrized deviations of Kerr spacetimes ("bumpy" or "quasi-Kerr" BHs), studying geodesics and EMRI waveforms. In some of these solutions, the deviation only affects the quadrupole moment [175, 178]; in others, higher-order multipoles are also affected [181187]. However, in many of these "bumpy" BH solutions the spacetime metric is not parametrized by multipole moments. These solutions have also been used to devise non-dynamical tests of the "no-hair" hypotheses based on astrophysical, electromagnetic observations (see Sec. 5.4).

\subsection{Tests with electromagnetic observations}

Non-dynamical tests of the "no-hair" hypothesis can also be done with astrophysical observations of the electromagnetic emission from the surroundings of $\mathrm{BH}$ candidates (see e.g. [188 190] and references therein).

5.4.1. Motion of stars A promising measurement of the multipole moments of $\mathrm{BH}$ candidates is the (electromagnetic) observation of stars on tight orbits around supermassive BHs, most specially Sgr A*, the compact object at the center of our galaxy [191 194]. Although such tests are complicated by several additional factors (related to the fact that such orbits have typical radii much larger than those involved in GW observations), progress in instrumentation makes them attractive possibilities in the near-future.

The idea is very simple, and consists on measuring the pericenter and orbital plane precession of stars orbiting a massive $\mathrm{BH}$, on tight and eccentric enough orbits. The precession depends on the mass of the central object (the Schwarzschild - $S$ - part of the geometry), on the spin $J$ and on the quadrupole moment $Q$ of the $\mathrm{BH}$ and can therefore be inverted to estimate each of these quantities, testing the no-hair hypothesis.

Define,

$$
\begin{aligned}
& A_{S}=\frac{6 \pi}{c^{2}} \frac{G M}{a\left(1-e^{2}\right)}, \\
& A_{J}=\frac{4 \pi j}{c^{3}}\left[\frac{G M}{a\left(1-e^{2}\right)}\right]^{3 / 2}, \\
& A_{Q}=\frac{3 \pi j^{2}}{c^{4}}\left[\frac{G M}{a\left(1-e^{2}\right)}\right]^{2},
\end{aligned}
$$

with $M$ the central, massive object, $j$ its dimensionless spin, $e$ the star's eccentricity and $a$ its semi-major axis. In the orbit-averaged approximation, and assuming that the orbiting stars are much lighter than the central object, stars experience an advance of 


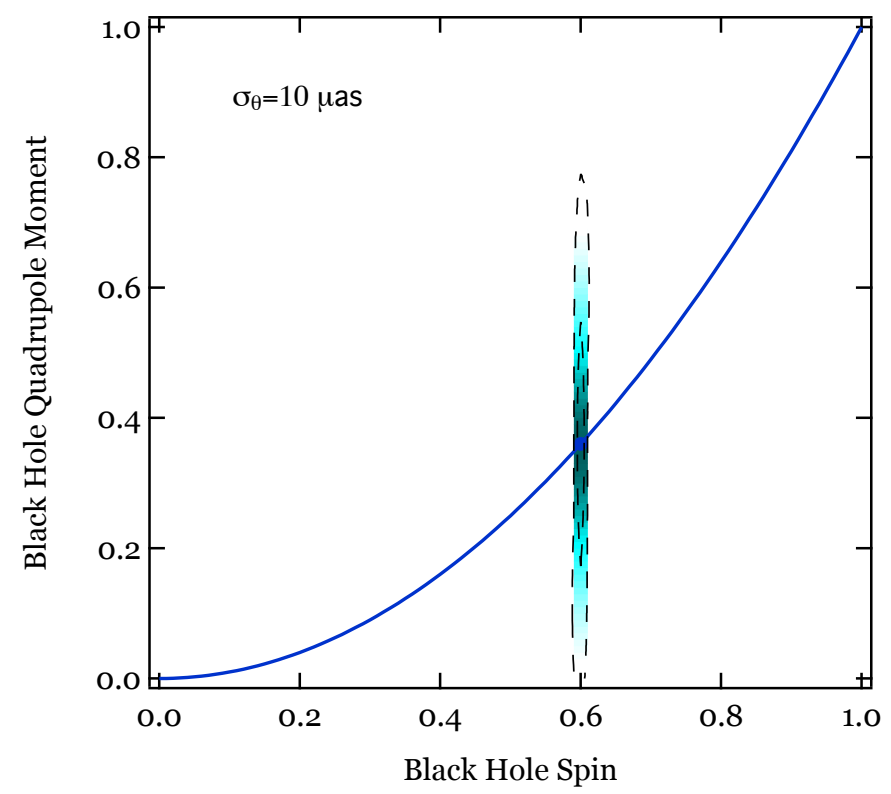

Figure 13. The posterior likelihood of measuring the spin and quadrupole moment of Sgr $\mathrm{A}^{*}$ by tracing the orbits of two stars with GRAVITY, assuming an astrometric precision of $10 \mu$ as. The dashed curves show the $68 \%$ and $95 \%$ confidence limits, while the solid curve shows the expected relation between these two quantities in the Kerr metric. The assumed (dimensionless) spin and quadrupole moment are $j=0.6$, $Q / M^{3}=0.36$ ). The two stars are assumed to have orbital separations equal to $800 \mathrm{M}$ and $1000 M$ and eccentricities of 0.9 and 0.8, respectively. Even at these relatively small orbital separations, tracing the orbits of stars primarily measures the spin of the BH, unless a very high level of astrometric precision is achieved. From Ref. [193].

the orbital periapse given by (to lowest post-Newtonian order)

$$
\delta \varpi=A_{S}-2 A_{J}-\frac{1}{2} A_{Q}\left(1-3 \cos ^{2} i\right),
$$

per orbit. In addition the orbital plane also precesses due to coupling between the orbital angular momentum and the central object spin $J$. The nodal precession is

$$
\delta \Omega=A_{J}-A_{Q} \cos i
$$

with $i$ the orbital inclination.

It can be seen that the Schwarzschild contribution exceeds the spin and quadrupole terms for in-plane precession, for most of the parameter space. Fortunately, the orbital plane precession depends only on the spin and quadrupole contributions. However, to test the no-hair hypothesis, one must determine five parameters (the $\mathrm{BH}$ mass, quadrupole moment and the three spin components), thus the orbits of two stars are required [191].

In addition, there are a number of effects that complicate the determination of the BH's multipole moments, such as 191-193.

i. The contribution of other orbiting stars to the mass, spin and quadrupole moment of the central object. This contribution can be estimated once a stellar density distribution 
is known or prescribed. Assuming a number density of stars

$$
n(a, \theta)=N_{0} / a_{0}^{3}\left(a / a_{0}\right)^{-2} n_{\theta}(\theta),
$$

one gets for the relative contributions to the mass, spin and quadrupole moment [192, 193 ,

$$
\begin{aligned}
\frac{\delta M}{M} & =4.8 \times 10^{-8} M_{6}\left(\frac{a_{0}}{1 \mathrm{pc}}\right)^{-1} a_{1}, \\
j \frac{\delta J}{J} & =3 \times 10^{-8} M_{6}\left(\frac{a_{0}}{1 \mathrm{pc}}\right)^{-1} a_{1}^{3 / 2}, \\
Q / M^{3} \frac{\delta Q}{Q} & =4 \times 10^{-10} M_{6} \frac{\tilde{n}_{\theta}}{0.1}\left(\frac{a_{0}}{1 \mathrm{pc}}\right)^{-1} a_{1}^{3} .
\end{aligned}
$$

Here, $a_{1} \equiv \frac{a}{M}, M_{6} \equiv \frac{M_{*}}{10^{6} M_{\odot}}, M_{*}$ being the total mass of stars inside a characteristic orbital separation $a_{0}$, and

$$
\tilde{n}_{\theta}=\int_{-1}^{1}\left(3 \cos ^{2} \theta-1\right) n_{\theta}(\theta) d \cos \theta,
$$

characterizes the angular distribution of stars. The strongest constraint here comes from the quadrupole corrections, which depend sensitively on the semi-major axis $a$. For the corrections to be under control, $a \lesssim 1000 M$.

ii. Perturbations to the orbit of the stars being measured. These effects include

iia. Decoherence of the orbit due to Newtonian interactions with other stars, on a timescale

$$
t_{N}=12.6 \times 10^{8} M_{6}^{-1 / 2}\left(\frac{a_{0}}{1 \mathrm{pc}}\right)^{-1 / 2} a_{1}\left(\frac{m_{*}}{M_{\odot}}\right)^{-1 / 2}\left(\frac{M}{4.3 \times 10^{6} M_{\odot}}\right)^{3 / 2} \mathrm{sec},
$$

where $m_{*}$ is the average mass of a star within the stellar cluster.

iib. Decoherence is the dominant perturbing effect, but in hydrodynamic interactions with the accretion flow (drag), stellar winds and tidal effects on the stars being measured introduce additional sources of error. These are quantified in Ref. [193].

In Ref. [193] the authors have studied how one can constrain the spin and quadrupole moment of Sgr A*, by measuring the motion of two stars on eccentric orbits. The results are shown in Fig. 13, assuming an astrometric precision of $10 \mu$ as. It turns out that, even at the small orbital separations used in their study, tracing the orbits of stars primarily measures the spin of the $\mathrm{BH}$.

We note, however, that even such large errors on the $\mathrm{BH}$ quadrupole moment already impose some constraints on some hairy BH solutions (see, e.g, Ref. [65], where some of the BH solutions have extremely large quadrupole moments).

5.4.2. Motion of pulsars It was pointed out some time ago that the observation of a single pulsar in orbit around a very compact object might still allow for tests of the Kerr hypothesis [195]. The basic idea rests, of course, on data available through pulsar timing. The strategy and sources of error are described in detail in Refs. [193, 196]. The mass of the central object (and the inclination of the orbital plane) can be determined from the 
precession of the periastron or via Shapiro delay. The Lense-Thirring precession, along with a measurement of the precession of periastron, the projected semi-major axis and their time derivatives, allow for the determination of all three spin components. Finally, Roemer delay can be used to estimate the quadrupole moment [196].

Tests of the Kerr hypothesis using single pulsars requires high-eccentricity pulsar and sub-year orbital periods, but is a promising tool for the near-future [193, 196].

5.4.3. Accretion disks Matter accretion onto $\mathrm{BHs}$ is one of the most luminous phenomena in the universe: the efficiency for converting rest mass into radiation in this process can be as large as $\sim 10 \%$, much larger than, i.e., thermonuclear processes. The radiation emitted by accreting matter at few gravitational radii from the horizon, can be a promising probe of the $\mathrm{BH}$ strong-field region.

The matter surrounding a BH forms an accretion disk [197, 198], in which each element approximately moves in circular, Keplerian orbits. As the matter element loses angular momentum, it moves inward to a different circular orbit, until it reaches the inner edge of the disk, corresponding to the ISCO of the BH spacetime. Then, since there is no stable orbit inside the ISCO, the matter dynamically falls into the BH. As the ISCO represents a transition point in the physics of the accretion disk, it should be possible to extract its location, $r_{I S C O}$, from the electromagnetic signal emitted (mostly in the X-ray band) by the accreting matter.

As noticed in [199], $r_{I S C O}$ (or, equivalently, the ISCO angular velocity $\Omega_{I S C O}$ ) is characterized by the first multipole moments of the central object. In particular, the contribution of the quadrupole moment to $r_{I S C O}$ is comparable to that of the $\mathrm{BH}$ spin. Therefore, a measurement of $r_{I S C O}$ or $\Omega_{I S C O}$, either for a stellar-mass $\mathrm{BH}$ or for a supermassive BH, would be a (non-dynamical) test of the "no-hair" hypothesis. In practice, since the physics of accretion disks is very complex, is not easy to extract $r_{I S C O}$ with good accuracy from the electromagnetic signal from accreting BHs. The two main approaches to extract information on the $\mathrm{BH}$ spacetime from the X-ray emission of the accretion disk are the analysis of the iron $K \alpha$ line [200], and the study of the thermal component of the spectrum using the so-called continuum-fitting method [201]. Presently, they allow to measure (with some confidence) the value of BH spins [202, 203], but the constraints on the BH spacetime (see e.g. [189, 190, and references therein) are still weak.

The iron $K \alpha$ line is the brightest component of the X-ray emission from the accretion disk of supermassive BHs, and one of the brightest components in the case of stellar-mass BHs. It is broadened and skewed due to (special and general) relativistic effects and Doppler effect, which determine a characteristic shape. An analysis of this shape (assuming that the spacetime is described by the Kerr metric) allows to measure the $\mathrm{BH}$ spin and the inclination of the accretion disk, even if the $\mathrm{BH}$ mass is unknown [203]. In order to test the $\mathrm{BH}$ spacetime, the theoretical model of the line shape has been extended to parametrized deviations of the Kerr spacetime ("quasiKerr" or "bumpy" BHs, see Sec. 5.3); a comparison of these models with future, more 
accurate observations of the iron $K \alpha$ line can allow to set bounds to the parameters characterizing these solutions, and then to test the "no-hair" hypothesis [204 211]. The main limitation of this approach is that current theoretical models of the iron line are too simple to prevent systematic effects, which may dominate possible deviations from Kerr spacetime [189].

The spectrum emitted by accretion disks of stellar-mass BHs has a strong thermal component, which can be analysed with the continuum-fitting method, i.e. by comparison with a theoretical model, in order to measure $r_{I S C O}$. In this model the standard, Novikov-Thorne description of of the accretion disk [197, 198] is assumed: the disk is geometrically thin, optically thick, orthogonal to the $\mathrm{BH}$ spin, with matter moving in circular geodesics, and inner edge at $r_{I S C O}$ (this description is believed to be accurate for a subset of the actual BH accretion disks). The background spacetime is assumed to be described by the Kerr metric. If the mass, distance and disk inclination are known from independent measurements, this approach allows to determine $r_{I S C O}$ and then to measure the BH spin [202]. This model has been generalized to "quasiKerr" or "bumpy" BH spacetimes, in order to set bounds, comparing the model with observational data, to the deviations from the Kerr metric, and then to test the "no-hair" hypothesis [204, 209 215]. While the continuum-fitting method can provide, assuming the Kerr background, a reliable measurement of the BH spin for a significant fraction of the stellar-mass BHs, it is much less effective in constraining deviations from the Kerr metric. Indeed, the parameters characterizing the deviation are typically degenerate with $r_{I S C O}$ [189, 190]. This degeneracy is partially removed when the ISCO is very close to the horizon, i.e. for near-extremal BHs.

5.4.4. Black hole "shadows" As discussed in Sec. 5.3, when matter is moving very close to a $\mathrm{BH}$ (or any compact object) a multipolar decomposition of the gravitational field is not particularly useful since all, or a substantial number of, multipoles contribute to the gravitational potential and to the motion of matter. However, the spacetime around compact objects possesses unique features - such as innermost stable circular orbits, light rings (unstable null geodesics), etc [216] - that might be used as smoking guns of the BHnature of the object and even of GR. In particular, the null geodesics carry information about the effective size of BHs, since in essence any particle or light ray penetrating the light ring will never reach asymptotic observers. Thus, BHs create "shadows" of matter around them [217 219]. The exact shape and appearance of BHs depends on the source illuminating them, but a crucial ingredient determining the optical appearance is the rotation rate, which determines how close to the horizon the co-rotating light ring is, and how far away from the horizon the counter-rotating light ring is. The rotation rate also determines how tight the accretion disk can bind to the $\mathrm{BH}$, and how much the gravitational and Doppler shift emitted from the disk will be. An example of the optical appearance of BHs is shown in Fig. 14. for a Kerr BH rotating with $a / M=0.9$ and being illuminated by an accretion torus 99 .

Observations of $\mathrm{BH}$ shadows as a tool to probe the geometry close to the event 

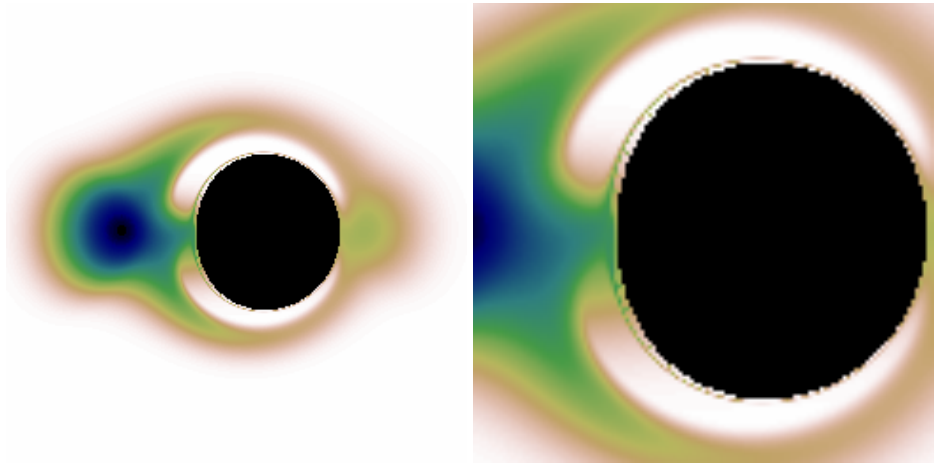

Figure 14. Image at $\lambda=1.3 \mathrm{~mm}$ of an accretion torus surrounding a Kerr $\mathrm{BH}$. The Kerr BH is spinning with $a / M=0.99$, and the torus is being observed at an angle $i=85^{\circ}$. The torus has an inner radius of $4.2 M$, temperature $5.3 \times 10^{10} \mathrm{~K}$ and a polytropic index $5 / 3$. For further details see Ref. [99. The color bar indicates the cgs value of specific intensity. The directions on the observer's sky that asymptotically approach the event horizon when ray tracing backwards in time are marked in black color. The black area at the center of the image is the BH shadow. Its exterior limit nearly coincides with the light ring. The right panel shows a zoom on the central region. From Ref. 99.
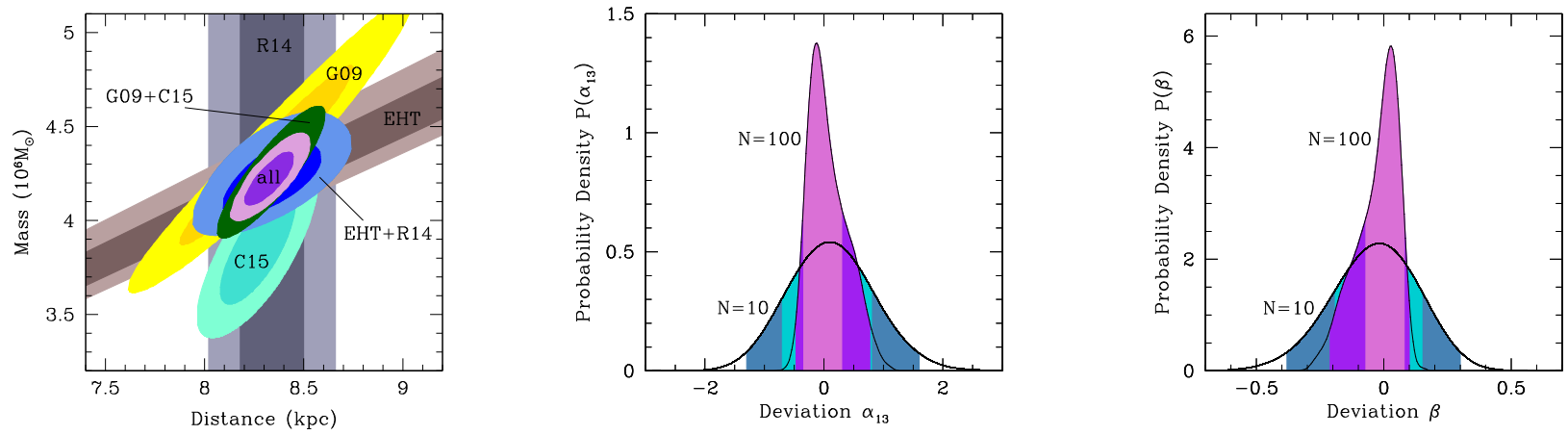

Figure 15. Left panel: $1 \sigma$ and $2 \sigma$ confidence contours of the probability density of the mass and distance of Sgr A* for existing measurements (S-stars, "G09" 220]; masers, "R14" 221]; star cluster, "C15" 222]), a simulated measurement of the shadow size of Sgr A* for $N=10$ observations with a seven-station EHT array ("EHT"), and several combinations thereof. The simulated EHT measurement improves the other constraints on the mass and distance significantly. Center and right panels: Simulated $1 \sigma$ and $2 \sigma$ confidence contours of the probability density of the deviation parameters $\alpha_{13}$ and $\beta$, respectively, corresponding to $N=10$ and $N=100$ EHT observations, each marginalized over the mass and distance using the combination of all data sets ("all") in the $N=10$ case and of simulated stellar-orbit observations from a 30m-class telescope 223 in the $N=100$ case. From Ref. 224]. 
horizon became possible with the advent of powerful instruments such as the Event Horizon Telescope [225]. The main obstacles to performing tests of GR with observations of $\mathrm{BH}$ shadows are (a) the large number of parameters that describe the shadow, including the inclination angle of the object, the mass and angular momentum of the $\mathrm{BH}$, and all the details of the accretion mechanism (the illuminating source); (b) the lack of a robust parametrization of deviations from the Kerr geometry, in the strong-field limit.

Recently, a proof-of-principle describing tests of the Kerr hypothesis with observations of $\mathrm{BH}$ shadows was put forward [224]. As a background, the following metric (belonging to the class of "quasi-Kerr" spacetimes introduced in [187]) was proposed,

$$
\begin{aligned}
d s^{2} & =-\frac{\Sigma\left(r^{2}-2 M r+a^{2} \cos ^{2} \theta\right)}{\left(A_{1}\left(r^{2}+a^{2}\right)-a^{2} \sin ^{2} \theta\right)^{2}} d t^{2}-\frac{2 a \sin ^{2} \theta \Sigma\left(A_{1}\left(r^{2}+a^{2}\right)-\tilde{\Delta}\right)}{\left(A_{1}\left(r^{2}+a^{2}\right)-a^{2} \sin ^{2} \theta\right)^{2}} d t d \phi \\
& +\frac{\Sigma}{\tilde{\Delta}} d r^{2}+\Sigma d \theta^{2}+\left[\frac{A_{1}^{2}\left(r^{2}+a^{2}\right)^{2}-a^{2} \tilde{\Delta} \sin ^{2} \theta}{\left(A_{1}\left(r^{2}+a^{2}\right)-a^{2} \sin ^{2} \theta\right)^{2}}\right] \Sigma \sin ^{2} \theta d \phi^{2}
\end{aligned}
$$

where $\tilde{\Delta}=\Delta+\beta M^{2}$ and $A_{1}=1+\alpha_{13} M^{3} / r^{3}$. This geometry is parametrized by the BH mass $M$, angular momentum $J=M a$ and the constants $\alpha_{13}, \beta$. We should point out that this particular form of the metric is rather ad-hoc and does not describe any known theory, but that in any case the results are to be taken only as proof-of-principle.

The shadow resulting from the metric (5.31) was compared to simulations of observations by the Event Horizon Telescope of the BH at the center of our galaxy, Sgr A*. The result is summarized in Fig. 15. The study uses a reconstructed image of Sgr $A^{*}$ based on a simulated image of a radiatively-inefficient accretion flow. Within the working hypothesis, the prospects for constraining the unknown parameters $\beta, \alpha_{13}$ are good: with 100 observations, the parameters can be determined to be

$$
\begin{aligned}
& \alpha_{13}=0.13_{-0.21-0.34}^{+0.43+0.9} \\
& \beta=0.03_{-0.10-0.24}^{+0.05} .0 .07
\end{aligned}
$$

The uncertainties associated with these parameters are such that they are effectively of order $\mathcal{O}(1)$, precisely the order at which they enter in the metris $\$$.

Hairy BHs arising naturally from simple theories, such as the minimally coupled scalar theory (3.6) [28, 65], will also give rise to shadows which can, in principle, be discriminated from those of Kerr BHs [227].

It should be noted that shadow observations can also, in principle, be used to test the BH-nature of compact objects. One could expect that objects without a horizon, and presumably with a surface, will feature a bright surface, allowing immediately to

$\S$ Note also that, when trying to translate such constraints to specific modified theories, care has to be exercised: in EDGB theory, for example, the natural parameter that appears in the equations is $\alpha^{\prime} / M^{2}$ where $\alpha^{\prime}$ is the theory's coupling constant, with dimensions of mass squared [27, 226]. Effectively then, one is constraining $\alpha^{\prime} / M^{2}$ which yields poor constraints on the coupling constant itself for supermassive BHs. 
discriminate between BHs and, say, gravastars. It was pointed out in Ref. [99] that the main factor determining the shadow is the light ring. As such, horizonless compact objects with a light ring can mimick Kerr BHs very efficintly, provided the illuminating source is not accreted towards the center of the object.

\section{Conclusions}

It is by now well-known that the Kerr family is not the most general solution of Einstein's field equations in the presence of reasonable forms of matter. However, there are good reasons to believe that BHs which do not belong to the Kerr family are either dynamically unstable or do not form out of realistic collapse scenarios.

The no-hair or Kerr hypothesis is therefore a cherished belief, which upcoming experiments can test, either in the GW or electromagnetic band. Some of these measurements will be more sensitive to the null geodesic around compact objects, than to the presence of the event horizon itself [116]... but given that even light rings are a unique feature of relativistic theories of gravity, these are all exciting years ahead.

Acknowledgments. We thank the anonymous referee for useful suggestions, which contributed to improve the quality of the manuscript. We are indebted to João Costa, Carlos Herdeiro, Chris Moore, Thomas Sotiriou, Norbert Wex, and Nicolas Yunes for useful comments on the manuscript. V. C thanks the Physics Department of the University of Rome "La Sapienza" for hospitality while this work was being completed. V.C. acknowledges financial support provided under the European Union's H2020 ERC Consolidator Grant "Matter and strong-field gravity: New frontiers in Einstein's theory" grant agreement no. MaGRaTh-646597, and FCT for Sabbatical Fellowship nr. SFRH/BSAB/105955/2014. Research at Perimeter Institute is supported by the Government of Canada through Industry Canada and by the Province of Ontario through the Ministry of Economic Development \& Innovation. This work was supported by the H2020-MSCA-RISE-2015 Grant No. StronGrHEP-690904.

\section{Multipole moments in Newtonian gravity and in general relativity}

In this Appendix we describe the multipole expansion framework, discussed in Section 5.1, both in Newtonian gravity and in GR. For a more detailed discussion, we refer the reader to [119, 120] (Newtonian gravity) and [121,123, 228] (GR). 
Multipole moments in Newtonian gravity

The Newtonian gravitational potential in the exterior of a massive body with density $\rho(t, \vec{x})$ is the solution of Poisson's equation $\nabla^{2} \Phi=4 \pi G \rho$ in vacuum:

$$
\Phi(t, \vec{x})=-G \int \frac{\rho\left(t, \vec{x}^{\prime}\right)}{\left|\vec{x}-\vec{x}^{\prime}\right|} d^{3} x^{\prime} .
$$

With a Taylor expansion of $1 /\left|\vec{x}-\vec{x}^{\prime}\right|$ around $\vec{x}^{\prime}=\overrightarrow{0}$ and a spherical harmonic decomposition, this solution can be written as a series in $1 / r$ (where $r=|\vec{x}|$ ), which is called multipolar expansion of the potential:

$$
\Phi(t, \vec{x})=-G \sum_{l m} \frac{1}{r^{l+1}} \frac{4 \pi}{2 l+1} I_{l m}(t) Y_{l m}(\theta, \phi),
$$

where $Y_{l m}$ are the usual spherical harmonics, and

$$
I_{l m}(t)=\int \rho(t, \vec{x}) r^{l} Y_{l m}(\theta, \phi) d^{3} x
$$

are the multipole moments of the potential.

This expansion can also be expressed in terms of symmetric-trace-free (STF) tensors [119, 123]:

$$
\Phi(t, \vec{x})=-G \sum_{l=0}^{\infty} \frac{1}{r^{l+1}} \frac{(2 l-1) ! !}{l !} I^{<i_{1} \cdots i_{l}>} n^{i_{1}} \cdots n^{i_{l}},
$$

where $(2 l-1) ! !=(2 l-1)(2 l-3)(2 l-5) \cdots 1, n^{i}=x^{i} / r$ (in polar coordinates, $\left.n^{i}=(\sin \theta \cos \phi, \sin \theta \sin \phi, \cos \theta)\right)$, the brackets $<\cdots>$ denote the symmetric and trace-free part of a tensor, and $I^{<i_{1} \cdots i_{l}>}(t)=\int \rho\left(t, \vec{x}^{\prime}\right)\left(x^{i_{1}} \cdots x^{i_{l}}\right.$ - trace parts $) d^{3} x$ are the multipole moments. For a given value of $l$, the components of the STF tensor $n^{<i_{1}} \cdots n^{i_{l}>}$ are combinations of the spherical harmonics $Y_{l m}$ with $m=-l, \ldots, l$, and $I^{<i_{1} \cdots i_{l}>}$ are combinations of the multipoles 6.3$) I_{l m}$. The first terms of this expansion are

$$
\Phi=-\frac{G M}{r}-\frac{3 G}{2} I^{<i j>} x^{i} x^{j}
$$

where $I=M$ is the mass, $I^{i}=\int \rho x^{i} d^{3} x=0$ in the center-of-mass frame, $I^{<i j>}=$ $\int \rho\left(x^{i} x^{i}-\delta^{i j} r^{2} / 3\right) d^{3} x$ is the quadrupole moment $\|$.

In most cases, one considers the multipole expansion of a stationary, axisymmetric body with symmetry axis $\hat{k}=(0,0,1)$. Then, the only non-vanishing moments are $I_{l 0}$ and, defining

$$
M_{l} \equiv \sqrt{\frac{4 \pi}{2 l+1}} I_{l 0}=\int \rho(\vec{x}) r^{l} P_{l}(\cos \theta) d^{3} x,
$$

the expansion (6.2) reduces to Eq. (5.1):

$$
\Phi(\vec{x})=-G \sum_{l=0}^{\infty} \frac{M_{l}}{r^{l+1}} P_{l}(\cos \theta),
$$

\| Some authors use a different normalization for the STF multipoles, i.e. $Q^{<i_{1} \cdots i_{l}>}=(2 l-1) ! ! I^{<i_{1} \cdots i_{l}>}$. With this notation the quadrupole tensor is $Q^{<i j>}=\int \rho\left(3 x^{i} x^{i}-\delta^{i j} r^{2}\right) d^{3} x$ as, e.g., in [119]. 
where, as discussed in Section 5.1, $M_{0}=M, M_{1}=0$ in the center-of-mass frame, $M_{2}=Q$ quadrupole moment. With the further assumption that the body (and then, the gravitational potential) is reflection-symmetric across the equatorial plane, i.e., symmetric for $\theta \rightarrow \pi-\theta$, then $M_{2 l+1}=0$ : the only non-vanishing multipoles are those with even values of $l$.

In STF notation, axi-symmetry with respect to $\hat{k}$ implies that $I^{\left.<i_{1} \cdots i_{l}\right\rangle} \propto$ $k^{<i_{1}} \cdots k^{i_{l}>}$. Using normalization properties of STF tensors [120], it can be shown that

$$
I^{<i_{1} \cdots i_{l}>}=M_{l} k^{<i_{1}} \cdots k^{i_{l}>} .
$$

For instance, the quadrupole STF tensor is $I^{<i j>}=M_{2}\left(k^{i} k^{j}-\delta^{i j} / 3\right)=$ $Q \operatorname{diag}(-1 / 3,-1 / 3,2 / 3)$.

\section{Multipole moments in general relativity: the Geroch-Hansen construction}

Geroch and Hansen developed a formalism to define and compute the multipole moments of stationary, asymptotically flat solutions of Einstein's equations in vacuum [121, 122]. Their approach has then been generalized to non-vacuum spacetimes, including the electromagnetic field [229] (see also [230]) and a scalar field [179]. The latter work can also be seen as a generalization to modified gravity theories: the multipole structure with a scalar field, derived in [179], also applies to vacuum spacetimes in BergmannWagoner scalar-tensor theories [231, 232]. Other scalar-tensor theories with additional terms falling off rapidly enough, such as EDGB theory [82], enjoy the same multipole structure, as can be seen by comparing the derivation of the quadrupole moment in EDGB BHs [180] with the computation of multipole moments in Bergmann-Wagoner theories [179].

In the Geroch-Hansen construction, multipole moments are introduced as tensors at infinity, generated by a set of potentials. The asymptotic behaviour is defined without introducing a specific coordinate frame, through an asymptotic completion of spacetime in which, after a conformal rescaling of the metric, the spacetime is extended to include the "infinite point" $\Lambda$. To understand this procedure, let us consider for simplicity the Euclidean flat space in Newtonian theory, with metric $d s^{2}=\delta^{i j} d x^{i} d x^{j}$. The multipole expansion of a potential $\Phi(\vec{x})$ is

$$
\Phi=\sum_{l \geq 0} \frac{1}{l !} \frac{1}{r^{l+1}} Q^{<i_{1} \cdots i_{l}>} x^{i_{1}} \cdots x^{i_{l}}=\frac{Q}{r}+\frac{Q^{i}}{r^{3}} x^{i}+\frac{1}{2} \frac{Q^{<i j>}}{r^{5}} x^{i} x^{j}+\ldots
$$

Changing coordinates to $\bar{x}^{i}=x^{i} / r^{2}$, the metric reads $d s^{2}=\left(\delta^{i j} d \bar{x}^{i} d \bar{x}^{j}\right) / \bar{r}^{4}$. With a conformal transformation the metric becomes $\overline{d s}^{2}=\Omega^{2} d s^{2}=\delta^{i j} d \bar{x}^{i} d \bar{x}^{j}$ (where $\Omega=\bar{r}^{2}$ ), regular at the infinite point $\Lambda(\bar{r}=0)$. In the new coordinate frame, the conformally rescaled field is

$$
\tilde{\Phi}\left(\bar{x}^{i}\right)=\Omega^{-1 / 2} \Phi=Q+Q^{i} \bar{x}^{i}+\frac{1}{2} Q^{<i j>} \bar{x}^{i} \bar{x}^{j}+\frac{1}{6} Q^{<i j k>} \bar{x}^{i} \bar{x}^{j} \bar{x}^{k}+\ldots
$$

The STF derivatives of $\tilde{\Phi}$ evaluated at the infinite point $\Lambda$ yield the multipole moments: $\tilde{\Phi}_{\mid \Lambda}=Q, \tilde{\Phi}_{, i \mid \Lambda}=Q^{i}, \tilde{\Phi}_{,<i j>\mid \Lambda}=Q^{<i j>}, \tilde{\Phi}_{,<i j k>\mid \Lambda}=Q^{<i j k>}$, and so on. 
In GR the procedure is the same, with some differences:

- The 3-space is the space of orbits of the timelike Killing vector (i.e., choosing the time variable corresponding to the Killing vector, any $t=$ const. submanifold). This is a curved space, with its own covariant derivative $D_{i}\left(\bar{D}_{i}\right.$ after the conformal transformation).

- The conformal transformation is not given explicitly; it is only required that the conformally-rescaled metric is smooth at the infinite point $\Lambda$, and that the conformal factor and its first derivative (but not the second) vanish in $\Lambda$, i.e. that $\Omega$ falls off "like $1 / r^{2}$ ". Actually, this requirement is the definition of "asymptotic flatness" of the spacetime.

- The derivatives on the fields are covariant derivatives $\bar{D}_{i}$. Moreover, the requirement that the moments do not depend on the choice of $\Omega$ determines extra terms involving the Ricci tensor (of the 3-space) in the definition of the moments. For instance, $Q^{<i j>}=\left(\bar{D}_{<i} \bar{D}_{j>} \Phi-1 / 2 \mathcal{R}_{<i j>} \Phi\right)_{\mid \Lambda}$.

In this way, the asymptotic expansion of the potentials can be constructed in a coordinate-independent way; the multipoles are defined as tensors in $\Lambda$.

The next step is to define the potentials corresponding to the multipoles of the four-dimensional spacetime. Given the Killing vector field $\xi^{\mu}$, the mass potential and the angular potential are $\Phi_{M}=\left(\lambda^{2}+\omega^{2}-1\right) /(4 \lambda)$ and $\Phi_{J}=\omega /(2 \lambda)$, where

$$
\begin{aligned}
\lambda & =-\xi^{\mu} \xi_{\mu} \\
\omega_{\alpha} & =\epsilon_{\alpha \beta \gamma \delta} \xi^{\beta} \xi^{\delta ; \gamma}=\omega_{, \alpha}
\end{aligned}
$$

(the fact that $\omega_{\alpha}$ is a gradient is a consequence of Einstein's field equations). The multipole expansion of these potentials, defined with the procedure outlined above, yields the mass multipoles $M^{\left.<i_{1} \cdots i_{l}\right\rangle}$ and the current multipoles $S^{\left.<i_{1} \cdots i_{l}\right\rangle}$. In the weak-field limit, the Geroch-Hansen mass multipole moments reduce to the Newtonian multipole moments $I^{<i_{1} \cdots i_{l}>}$ defined in Eq. (6.4), with a different normalization: $M^{<i_{1} \cdots i_{l}>}=(2 l-1) ! ! I^{<i_{1} \cdots i_{l}>}$ (STF mass monopoles in Thorne's paper [123], instead, have the same normalization as the Newtonian multipole moments).

When extra fields are present, they correspond to new potentials and then to new sets of multipole moments. For instance, a scalar field $\phi$ brings a potential $\Phi_{S}=\phi[179]$ which yields a set of scalar multipoles $S^{\left\langle i_{1} \cdots i_{l}\right\rangle}$. It should be noted that the Ricci terms $\mathcal{R}_{i j}$ in the definition of multipole moments induce a mixing of the moments associated to different fields.

When the spacetime is symmetric with respect to an axis $\hat{k}$,

$$
\begin{aligned}
M^{<i_{1} \cdots i_{l}>} & =(2 l-1) ! ! M_{l} k^{<i_{1}} \cdots k^{i_{l}>} \\
S^{<i_{1} \cdots i_{l}>} & =(2 l-1) ! ! S_{l} k^{<i_{1}} \cdots k^{i_{l}>} .
\end{aligned}
$$

The mass and current STF moments reduce to two sets of scalar moments $M_{l}, S_{l}$. The Geroch-Hansen procedure can be cast in a simpler form [124], exploiting the powerful Ernst potential formalism. Indeed, it can be shown that the complex combination 
$\zeta=\Phi_{M}+\mathrm{i} \Phi_{J}$ is the (secondary) Ernst potential, and Einstein's equations can be formulated in terms of the Ernst potential.

\section{Multipole moments in general relativity: Thorne's expansion}

The multipole moments of a stationary, isolated object can also be defined in terms of the asymptotic behaviour of the spacetime metric. Indeed, as shown by Thorne [123], the (asymptotic) spacetime metric can be expanded in inverse powers of a suitable radial coordinate; the coefficients of this expansion can be interpreted as the multipole moments:

$$
\begin{aligned}
g_{00}= & -1+\frac{2 M}{r}+\sum_{l \geq 2} \frac{1}{r^{l+1}}\left(\frac{2}{l !} M^{<a_{1} \cdots a_{l}>} n^{a_{1}} \cdots n^{a_{l}}+\left(l^{\prime}<l \text { harmonics }\right)\right) \\
g_{0 j}= & -2 \sum_{l \geq 1} \frac{1}{r^{l+1}}\left(\frac{1}{l !} \epsilon^{j k a_{l}} S^{<k a_{1} \ldots a_{l-1}>} n^{<a_{1}} \cdots n^{a_{l}>}+\left(l^{\prime}<l \text { harmonics }\right)\right. \\
& \left.+\left(l-\text { harmonics with parity }(-1)^{l}\right)\right) .
\end{aligned}
$$

The coordinate systems in which the spacetime metric has the form 6.13 are called ACMC coordinates: they are asymptotically cartesian and (when the source is weakfield and covered by the same coordinate system) the origin of the coordinate system lies at the center of mass of the source. It has been shown [139] that the definitions of multipole moments by Thorne and by Geroch-Hansen are equivalent. Actually, the moments appearing in [123] have different normalization; however, for simplicity of notation, in Eqns. (6.13) (and throughout this Review) we have used the normalizations of Geroch-Hansen, which are also those adopted in most of the recent literature on the subject.

As discussed above, when the spacetime is symmetric with respect to an axis $\hat{k}$, the multipole moments $M^{<a_{1} \cdots a_{l}>}, S^{<a_{1} \cdots a_{l}>}$ reduce to the scalar quantities $M_{l}$, $S_{l}$ (see Eqns. (6.12)). Using the normalization properties of STF tensors [120, 233], it can be shown that

$$
\begin{aligned}
& M^{<a_{1} \cdots a_{l}>} n^{<a_{1}} \cdots n^{a_{l}>} \quad=l ! M_{l} P_{l}(\cos \theta) \\
& \epsilon^{j k a_{l}} S^{<k a_{1} \ldots a_{l-1}>} n^{<a_{1}} \cdots n^{a_{l}>}=(l-1) ! \epsilon^{i j k} n^{i} k^{k} S_{l} P_{l}^{\prime}(\cos \theta)
\end{aligned}
$$

where $\cos \theta=\hat{n} \cdot \hat{k}$, and primes denote derivatives with respect to $\cos \theta$. The metric expansion can then be written as

$$
\begin{aligned}
& g_{00}=-1+\frac{2 M}{r}+\sum_{l \geq 2} \frac{1}{r^{l+1}}\left(M_{l} P_{l}(\cos \theta)+\left(l^{\prime}<l \text { harmonics }\right)\right) \\
& g_{0 j}=-2 \sum_{l \geq 1} \frac{1}{r^{l+1}}\left(\epsilon^{i j k} n^{i} k^{k} \frac{S_{l}}{l} P_{l}^{\prime}(\cos \theta)+\left(l^{\prime}<l \text { harmonics }\right)\right)
\end{aligned}
$$

(note that when the spacetime is symmetric with respect to the axis $\hat{k}$, the vector harmonics with parity $(-1)^{l}$ in the expansion of $g_{0 j}$ - see Eq. (6.13) - identically 
vanish). In polar coordinates $\hat{k}=(0,0,1), \hat{n}=(\sin \theta \cos \phi, \sin \theta \sin \phi, \cos \theta)$ and $\epsilon^{i j k} n^{i} k^{k} d x^{j}=r \sin ^{2} \theta d \phi$, therefore Eqns. 6.15) yield Eqns. (5.5).

If the source can be covered by de Donder coordinates (in which $\bar{h}^{\mu \nu} \equiv$ $-(-g)^{1 / 2} g^{\mu \nu}+\eta^{\mu \nu}$ satisfies $\bar{h}_{, \nu}^{\mu \nu}=0$, and Einstein's equations can be written as flatspace wave equations for $\bar{h}^{\mu \nu}$ ), it is possible to define an "effective" stress-energy tensor $\tau^{\mu \nu}$, which is the source of the wave equations. In these coordinates, Thorne's multipole moments can be expressed as integrals of $\tau^{\mu \nu}$ over the source [123]:

$$
\begin{aligned}
M^{<a_{1} \cdots a_{l}>} & =(2 l-1) ! ! \int \tau^{00} x^{<a_{1}} \cdots x^{a_{l}>} d^{3} x \\
S^{<a_{1} \cdots a_{l}>} & =\frac{2 l(2 l-1) ! !}{l+1} \int x^{i} \tau^{0 j} \epsilon^{i j<a_{l}} x^{a_{1}} \cdots x^{a_{l-1}>} d^{3} x .
\end{aligned}
$$

When the spacetime is symmetric with respect to an axis $\hat{k}$, Eqns. 6.16 give

$$
\begin{aligned}
& M_{l}=\int \tau^{00} r^{l} P_{l}(\cos \theta) d^{3} x \\
& S_{l}=\frac{2}{l+1} \int \epsilon^{i j k} \tau^{0 j} n^{i} k^{k} r^{l} P_{l}^{\prime}(\cos \theta) d^{3} x .
\end{aligned}
$$

In the case of a weak-field source, $\tau^{00}=\rho, \tau^{0 j}=\rho v^{j}$, and Eqns. (6.17) reduce to 141,142 ,

$$
\begin{aligned}
M_{l} & =\int \rho r^{l} P_{l}(\cos \theta) d^{3} x \\
S_{l} & =\frac{2}{l+1} \int \rho v^{\phi} r^{l} P_{l}^{\prime}(\cos \theta) \sin ^{2} \theta d^{3} x .
\end{aligned}
$$

\section{References}

[1] Abbott B P et al. (Virgo, LIGO Scientific) 2016 Phys. Rev. Lett. 116061102 (Preprint 1602.03837)

[2] Berti E, Cardoso V and Starinets A O 2009 Class. Quant. Grav. 26163001 (Preprint 0905.2975)

[3] Zerilli F J 1970 Phys. Rev. D2 2141-2160

[4] Kerr R P 1963 Phys. Rev. Lett. 11 237-238

[5] Newman E T, Couch R, Chinnapared K, Exton A, Prakash A and Torrence R 1965 J. Math. Phys. 6 918-919

[6] Bekenstein J D 1997 Black hole hair: 25 - years after Proceedings of the Second International A.D. Sakharov Conference on Physics: Moscow, Russia 20-24 May 1996 ed Dremin I M and Semikhatov A M (World Scientific) ISBN 9810228619 (Preprint gr-qc/9605059)

[7] Carter B 1999 Has the black hole equilibrium problem been solved? Proceedings, 8th Marcel Grossmann meeting, Jerusalem, Israel, June 22-27, 1997. ed Piran T (World Scientific) pp 136-155 ISBN 9810237936 (Preprint gr-qc/9712038) 
[8] Chrusciel P T, Costa J L and Heusler M 2012 Living Rev. Relativ. 157 (Preprint 1205.6112)

[9] Robinson D 1975 Phys. Rev. Lett. 34 905-906

[10] Robinson D 2009 Four decades of black holes uniqueness theorems (Cambridge University Press)

[11] Barausse E, Cardoso V and Pani P 2014 Phys. Rev. D89 104059 (Preprint 1404.7149)

[12] Cardoso V, Macedo C F B, Pani P and Ferrari V 2016 JCAP 1605054 (Preprint 1604.07845)

[13] Ionescu A D and Klainerman S 2011 (Preprint 1108.3575)

[14] Ionescu A and Klainerman S 2015 (Preprint 1501.01587)

[15] Dafermos M and Rodnianski I 2013 Clay Math.Proc. 17 97-205 (Preprint 0811. 0354)

[16] Hartle J B 1971 Phys. Rev. D3 2938-2940

[17] Teitelboim C 1972 Lett. Nuovo Cim. 3S2 397-400 [Lett. Nuovo Cim.3,397(1972)]

[18] Teitelboim C 1972 Lett. Nuovo Cim. 3S2 326-328 [Lett. Nuovo Cim.3,326(1972)]

[19] Teitelboim C 1972 Phys. Rev. D5 2941-2954

[20] Witek H, Cardoso V, Ishibashi A and Sperhake U 2013 Phys. Rev. D87 043513 (Preprint 1212.0551)

[21] Bekenstein J D 1972 Phys. Rev. D5 2403-2412

[22] Hawking S W 1972 Commun. Math. Phys. 25 167-171

[23] Sotiriou T P and Faraoni V 2012 Phys. Rev. Lett. 108081103 (Preprint 1109. 6324)

[24] Graham A A H and Jha R 2014 Phys. Rev. D90 041501 (Preprint 1407.6573)

[25] Berti E, Cardoso V, Gualtieri L, Horbatsch M and Sperhake U 2013 Phys. Rev. D87 124020 (Preprint 1304.2836)

[26] Heusler M 1996 Helv. Phys. Acta 69 501-528 (Preprint gr-qc/9610019)

[27] Berti E et al. 2015 Class. Quant. Grav. 32243001 (Preprint 1501.07274)

[28] Herdeiro C A R and Radu E 2015 Int. J. Mod. Phys. D24 1542014 (Preprint 1504.08209)

[29] Volkov M S 2016 (Preprint 1601.08230)

[30] Rhoades Clifford E J and Ruffini R J 1974 Phys. Rev. Lett. 32 324-327

[31] Berti E et al. 2007 Phys. Rev. D76 064034 (Preprint gr-qc/0703053)

[32] Sperhake U, Cardoso V, Pretorius F, Berti E and Gonzalez J A 2008 Phys. Rev. Lett. 101161101 (Preprint 0806.1738)

[33] Davis M, Ruffini R, Press W H and Price R H 1971 Phys. Rev. Lett. 27 1466-1469

[34] Baiotti L, Giacomazzo B and Rezzolla L 2008 Phys. Rev. D78 084033 (Preprint 0804.0594 ) 
[35] Vishveshwara C V 1970 Nature 227936

[36] Cardoso V, Miranda A S, Berti E, Witek H and Zanchin V T 2009 Phys. Rev. D79 064016 (Preprint 0812.1806)

[37] Webpage with Mathematica notebooks and numerical quasinormal mode Tables: http://centra.tecnico.ulisboa.pt/network/grit/files/ http://www.phy.olemiss.edu/ berti/ringdown/

[38] Berti E, Cardoso V and Will C M 2006 Phys. Rev. D73 064030 (Preprint gr-qc/0512160)

[39] Price R H 1972 Phys. Rev. D5 2419-2438

[40] Ching E S C, Leung P T, Suen W M and Young K 1995 Phys. Rev. D52 2118-2132 (Preprint $\mathrm{gr}-\mathrm{qc} / 9507035)$

[41] Frauendiener J, Hoenselaers C and Konrad W 1990 Classical and Quantum Gravity $7585-587$

[42] Brady P R, Louko J and Poisson E 1991 Phys. Rev. D44 1891-1894

[43] Vogt D and Letelier P S 2010 Mon. Not. Roy. Astron. Soc. 4021313 (Preprint 0911.4822 )

[44] Vogt D and Letelier P S 2010 Mon. Not. Roy. Astron. Soc. 406 2689-2700 (Preprint 1006.2280)

[45] Brown J D and Husain V 1997 Int. J. Mod. Phys. D6 563-573 (Preprint gr-qc/9707027)

[46] Rezzolla L, Baiotti L, Giacomazzo B, Link D and Font J A 2010 Class. Quant. Grav. 27114105 (Preprint 1001.3074)

[47] Sotiriou T P 2015 Class. Quant. Grav. 32214002 (Preprint 1505.00248)

[48] Bizon P 1990 Phys. Rev. Lett. 64 2844-2847

[49] Volkov M S and Galtsov D V 1990 Sov. J. Nucl. Phys. 51 747-753 [Yad. Fiz.51,1171(1990)]

[50] Choptuik M W, Hirschmann E W and Marsa R L 1999 Phys. Rev. D60 124011 (Preprint gr-qc/9903081)

[51] Bizon P, Chmaj T and Rostworowski A 2007 Class. Quant. Grav. 24 F55-F63 (Preprint 0704.0993)

[52] Bizon P and Chmaj T 1992 Phys. Lett. B297 55-62

[53] Droz S, Heusler M and Straumann N 1991 Phys. Lett. B268 371-376

[54] Heusler M, Droz S and Straumann N 1991 Phys. Lett. B271 61-67

[55] Greene B R, Mathur S D and O'Neill C M 1993 Phys. Rev. D47 2242-2259 (Preprint hep-th/9211007)

[56] Torii T, Maeda K I and Tachizawa T 1995 Phys. Rev. D51 1510-1524 (Preprint gr-qc/9406013) 
[57] Brito R, Cardoso V and Pani P 2015 Lect. Notes Phys. 906 pp.1-237 (Preprint 1501.06570)

[58] Damour T, Deruelle N and Ruffini R 1976 Lett.Nuovo Cim. 15 257-262

[59] Detweiler S L 1980 Phys.Rev. D22 2323-2326

[60] Cardoso V and Yoshida S 2005 JHEP 0507009 (Preprint hep-th/0502206)

[61] Dolan S R 2007 Phys.Rev. D76 084001 (Preprint 0705.2880)

[62] Okawa H, Witek H and Cardoso V 2014 Phys. Rev. D89 104032 (Preprint 1401.1548)

[63] Zilhão M, Witek H and Cardoso V 2015 Class. Quant. Grav. 32234003 (Preprint 1505.00797)

[64] Brito R, Cardoso V and Pani P 2015 Class. Quant. Grav. 32134001 (Preprint 1411.0686)

[65] Herdeiro C A R and Radu E 2014 Phys. Rev. Lett. 112221101 (Preprint 1403.2757)

[66] Herdeiro C, Radu E and Runarsson H 2016 (Preprint 1603.02687)

[67] Pani P, Cardoso V, Gualtieri L, Berti E and Ishibashi A 2012 Phys. Rev. D86 104017 (Preprint 1209.0773)

[68] Pani P, Cardoso V, Gualtieri L, Berti E and Ishibashi A 2012 Phys. Rev. Lett. 109131102 (Preprint 1209.0465)

[69] Kleihaus B, Kunz J and Yazadjiev S 2015 Phys. Lett. B744 406-412 (Preprint 1503.01672)

[70] Herdeiro C A R, Radu E and Rúnarsson H 2015 Phys. Rev. D92 084059 (Preprint 1509.02923)

[71] De Rujula A, Glashow S L and Sarid U 1990 Nucl. Phys. B333 173

[72] Perl M L and Lee E R 1997 Am. J. Phys. 65 698-706

[73] Holdom B 1986 Phys. Lett. B166 196

[74] Sigurdson K, Doran M, Kurylov A, Caldwell R R and Kamionkowski M 2004 Phys. Rev. D70 083501 [Erratum: Phys. Rev.D73,089903(2006)] (Preprint astro-ph/ 0406355)

[75] Davidson S, Hannestad S and Raffelt G 2000 JHEP 05003 (Preprint hep-ph/ 0001179)

[76] McDermott S D, Yu H B and Zurek K M 2011 Phys. Rev. D83 063509 (Preprint 1011.2907)

[77] Jacobson T 1999 Phys. Rev. Lett. 83 2699-2702 (Preprint astro-ph/9905303)

[78] Babichev E and Charmousis C 2014 JHEP 08106 (Preprint 1312.3204)

[79] Yagi K and Stein L C 2016 Class. Quant. Grav. 33054001 (Preprint 1602.02413)

[80] Alexander S and Yunes N 2009 Phys. Rept. 480 1-55 (Preprint 0907.2562) 
[81] Mignemi S and Stewart N R 1993 Phys. Rev. D47 5259-5269 (Preprint hep-th/ 9212146)

[82] Kanti P, Mavromatos N, Rizos J, Tamvakis K and Winstanley E 1996 Phys. Rev. D54 5049-5058 (Preprint hep-th/9511071)

[83] Yunes N and Pretorius F 2009 Phys. Rev. D79 084043 (Preprint 0902.4669)

[84] Konno K, Matsuyama T and Tanda S 2009 Prog. Theor. Phys. 122 561-568 (Preprint 0902.4767)

[85] Sotiriou T P and Zhou S Y 2014 Phys. Rev. Lett. 112251102 (Preprint 1312.3622)

[86] Cardoso V, Carucci I P, Pani P and Sotiriou T P 2013 Phys. Rev. Lett. 111111101 (Preprint 1308.6587)

[87] Cardoso V, Carucci I P, Pani P and Sotiriou T P 2013 Phys. Rev. D88 044056 (Preprint 1305.6936)

[88] Brito R, Cardoso V and Pani P 2013 Phys. Rev. D88 064006 (Preprint 1309.0818)

[89] Babichev E and Brito R 2015 Class. Quant. Grav. 32154001 (Preprint 1503. 07529)

[90] Barausse E and Sotiriou T P 2013 Class. Quant. Grav. 30244010 (Preprint 1307.3359)

[91] Hawking S W, Perry M J and Strominger A 2016 (Preprint 1601.00921)

[92] Gubser S S 2005 Class. Quant. Grav. 22 5121-5144 (Preprint hep-th/0505189)

[93] Jetzer P 1992 Phys. Rept. 220 163-227

[94] Schunck F E and Mielke E W 2003 Class. Quant. Grav. 20 R301-R356 (Preprint 0801.0307 )

[95] Liebling S L and Palenzuela C 2012 Living Rev. Rel. 156 (Preprint 1202.5809)

[96] Macedo C F B, Pani P, Cardoso V and Crispino L C B 2013 Phys. Rev. D88 064046 (Preprint 1307.4812 )

[97] Brito R, Cardoso V, Herdeiro C A R and Radu E 2016 Phys. Lett. B752 291-295 (Preprint 1508.05395)

[98] Brito R, Cardoso V, Macedo C F B, Okawa H and Palenzuela C 2016 Phys. Rev. D93 044045 (Preprint 1512.00466)

[99] Vincent F H, Meliani Z, Grandclement P, Gourgoulhon E and Straub O 2016 Class. Quant. Grav. 33105015 (Preprint 1510.04170)

[100] Mazur P O and Mottola E 2004 Proc. Nat. Acad. Sci. 101 9545-9550 (Preprint gr-qc/0407075)

[101] Gimon E G and Horava P 2009 Phys. Lett. B672 299-302 (Preprint 0706.2873)

[102] Damour T and Solodukhin S N 2007 Phys. Rev. D76 024016 (Preprint 0704.2667)

[103] Dzhunushaliev V, Folomeev V, Kleihaus B and Kunz J 2016 (Preprint 1601. 04124) 
[104] Broderick A E and Narayan R 2007 Class. Quant. Grav. 24 659-666 (Preprint gr-qc/0701154)

[105] Broderick A E and Narayan R 2006 Astrophys. J. 638 L21-L24 (Preprint astro-ph/0512211)

[106] Okawa H, Cardoso V and Pani P 2014 Phys. Rev. D89 041502 (Preprint 1311.1235)

[107] Yunes N, Yagi K and Pretorius F 2016 (Preprint 1603.08955)

[108] Straumann N and Zhou Z H 1990 Phys. Lett. B243 33-35

[109] Herdeiro C and Radu E 2015 Class. Quant. Grav. 32144001 (Preprint 1501. 04319)

[110] Cardoso V, Pani P, Cadoni M and Cavaglia M 2008 Phys. Rev. D77 124044 (Preprint 0709.0532)

[111] Pani P, Cardoso V, Cadoni M and Cavaglia M 2009 [PoSBHGRS,027(2008)] (Preprint 0901.0850)

[112] Pani P, Barausse E, Berti E and Cardoso V 2010 Phys. Rev. D82 044009 (Preprint 1006.1863)

[113] Keir J 2016 Class. Quant. Grav. 33135009 (Preprint 1404.7036)

[114] Cardoso V, Crispino L C, Macedo C F, Okawa H and Pani P 2014 Phys. Rev. D90 044069 (Preprint 1406.5510)

[115] Nunez D, Quevedo H and Sudarsky D 1996 Phys. Rev. Lett. 76 571-574 (Preprint gr-qc/9601020)

[116] Cardoso V, Franzin E and Pani P 2016 Phys. Rev. Lett. 116171101 (Preprint 1602.07309)

[117] Psaltis D, Perrodin D, Dienes K R and Mocioiu I 2008 Phys. Rev. Lett. 100091101 [Phys. Rev. Lett.100,119902(2008)] (Preprint 0710.4564)

[118] Barausse E and Sotiriou T P 2008 Phys. Rev. Lett. 101099001 (Preprint $0803.3433)$

[119] Jackson J D 1999 Classical electrodynamics (Wiley)

[120] Poisson E and Will C M 2014 Gravity: Newtonian, Post-Newtonian, Relativistic (Cambridge University Press)

[121] Geroch R P 1970 J. Math. Phys. 11 2580-2588

[122] Hansen R O 1974 J. Math. Phys. 15 46-52

[123] Thorne K S 1980 Rev. Mod. Phys. 52 299-339

[124] Fodor G, Hoensealers C and Perjés Z 1989 J. Math. Phys. 30 2252-2257

[125] Tapley B D, Bettadpur S, Watkins M and Reigber C 2004 Geophysical Research Letters 31

[126] Drinkwater M, Floberghagen R, Haagmans R, Muzi D and Popescu A 2003 Goce: Esa's first earth explorer core mission Earth gravity field from space-From sensors to earth sciences (Springer) pp 419-432 
[127] Ciufolini I, Paolozzi A and Paris C 2012 Overview of the lares mission: orbit, error analysis and technological aspects Journal of Physics: Conference Series vol 354 (IOP Publishing) p 012002

[128] Chmap URL http://science.nasa.gov/missions/champ/

[129] Grace URL http://www.csr.utexas.edu/grace/

[130] Gfz URL http://www.gfz-potsdam.de/en/home/

[131] Nasa URL http://www.nasa.gov/

[132] Dlr URL http://www.dlr.de/dlr/en/desktopdefault.aspx/tabid-10002/

[133] Beig R and Simon W 1980 Communications in Mathematical Physics 78 75-82

[134] Beig R and Simon W 1981 Proceedings of the Royal Society of London Series A $376333-341$

[135] Kundu P 1981 Journal of Mathematical Physics 22 2006-2011

[136] Backdahl T and Herberthson M 2005 Class. Quant. Grav. 22 1607-1621 (Preprint gr-qc/0502012)

[137] Backdahl T 2007 Class. Quant. Grav. 24 2205-2215 (Preprint gr-qc/0612043)

[138] Misner C, Thorne K and Wheeler J 1973 Gravitation (San Francisco: W. H. Freeman) ISBN 9780716703440

[139] Gürsel Y 1983 General relativity and gravitation 15 737-754

[140] Mathews J 1962 Journal of the Society for Industrial and Applied Mathematics $10768-780$

[141] Ryan F D 1997 Phys. Rev. D55 6081-6091

[142] Stein L C, Yagi K and Yunes N 2014 Astrophys. J. 78815 (Preprint 1312.4532)

[143] Ryan F D 1995 Phys. Rev. D52 5707-5718

[144] Laarakkers W G and Poisson E 1999 Astrophys. J. 512 282-287 (Preprint gr-qc/9709033)

[145] Berti E and Stergioulas N 2004 Mon. Not. Roy. Astron. Soc. 3501416 (Preprint gr-qc/0310061)

[146] Pappas G and Apostolatos T A 2012 Phys. Rev. Lett. 108231104 (Preprint 1201.6067)

[147] Yagi K, Kyutoku K, Pappas G, Yunes N and Apostolatos T A 2014 Phys. Rev. D89 124013 (Preprint 1403.6243)

[148] Pani P, Gualtieri L, Maselli A and Ferrari V 2015 Phys. Rev. D92 024010 (Preprint 1503.07365)

[149] Pani P, Gualtieri L and Ferrari V 2015 Phys. Rev. D92 124003 (Preprint 1509.02171)

[150] Ayzenberg D and Yunes N 2014 Phys. Rev. D90 044066 [Erratum: Phys. Rev.D91,no.6,069905(2015)] (Preprint 1405.2133) 
[151] Benhar O, Ferrari V, Gualtieri L and Marassi S 2005 Phys. Rev. D72 044028 (Preprint gr-qc/0504068)

[152] Pappas G and Apostolatos T A 2012 (Preprint 1211.6299)

[153] Hessels J, Ransom S, Stairs I, Freire P, Kaspi V and Camilo F 2006 Science (New York, NY) 3111901

[154] Akmal A, Pandharipande V R and Ravenhall D G 1998 Phys. Rev. C58 1804-1828 (Preprint nucl-th/9804027)

[155] Pitjeva E 2005 Solar System Research 39 176-186

[156] Pani P 2015 Phys. Rev. D92 124030 (Preprint 1506.06050)

[157] Weinstein L A and Zubakhov V B 1962 Extraction of signals from noise (PrenticeHall)

[158] Scharf L L 1991 Statistical Signal Processing, Detection, Estimation, and Time Series Analysis (Addison-Wesley)

[159] Flanagan E E and Hughes S A 1998 Phys. Rev. D57 4535-4565 (Preprint gr-qc/9701039)

[160] Berti E, Cardoso J, Cardoso V and Cavaglia M 2007 Phys. Rev. D76 104044 (Preprint 0707.1202)

[161] Gossan S, Veitch J and Sathyaprakash B S 2012 Phys. Rev. D85 124056 (Preprint 1111.5819)

[162] Meidam J, Agathos M, Van Den Broeck C, Veitch J and Sathyaprakash B S 2014 Phys. Rev. D90 064009 (Preprint 1406.3201)

[163] Ghosh A et al. 2016 (Preprint 1602.02453)

[164] Kamaretsos I, Hannam M and Sathyaprakash B 2012 Phys. Rev. Lett. 109141102 (Preprint 1207.0399)

[165] Kamaretsos I, Hannam M, Husa S and Sathyaprakash B S 2012 Phys. Rev. D85 024018 (Preprint 1107.0854 )

[166] Berti E, Sesana A, Barausse E, Cardoso V and Belczynski K 2016 (Preprint 1605.09286)

[167] Blázquez-Salcedo J L, Macedo C F B, Cardoso V, Ferrari F, Gualtieri L, Khoo F S, Kunz J and Pani P 2016 in preparation

[168] Barausse E, Cardoso V and Pani P 2015 J. Phys. Conf. Ser. 610012044 (Preprint 1404.7140)

[169] Nakamura T, Nakano H and Tanaka T 2016 Phys. Rev. D93 044048 (Preprint 1601.00356)

[170] Kinugawa T, Nakano H and Nakamura T 2016 PTEP 2016 031E01 (Preprint 1601.07217)

[171] Nakamura T and Nakano H 2016 PTEP $2016041 \mathrm{E} 01$ (Preprint 1602.02385)

[172] Fujita R 2012 Prog. Theor. Phys. 128 971-992 (Preprint 1211.5535) 
[173] Seoane P A et al. (eLISA) 2013 (Preprint 1305.5720)

[174] Aasi J et al. (VIRGO, LIGO Scientific) 2013 [Living Rev. Rel.19,1(2016)] (Preprint 1304.0670)

[175] Collins N A and Hughes S A 2004 Phys. Rev. D69 124022 (Preprint gr-qc/ 0402063)

[176] Ryan F D 1997 Phys. Rev. D56 1845-1855

[177] Barack L and Cutler C 2007 Phys. Rev. D75 042003 (Preprint gr-qc/0612029)

[178] Glampedakis K and Babak S 2006 Class. Quant. Grav. 23 4167-4188 (Preprint gr-qc/0510057)

[179] Pappas G and Sotiriou T P 2015 Phys. Rev. D91 044011 (Preprint 1412.3494)

[180] Kleihaus B, Kunz J and Mojica S 2014 Phys. Rev. D90 061501 (Preprint 1407.6884 )

[181] Manko V and Novikov I D 1992 Classical and Quantum Gravity 92477

[182] Gair J R, Li C and Mandel I 2008 Phys. Rev. D77 024035 (Preprint 0708.0628)

[183] Johannsen T and Psaltis D 2011 Phys. Rev. D83 124015 (Preprint 1105.3191)

[184] Vigeland S J and Hughes S A 2010 Phys. Rev. D81 024030 (Preprint 0911.1756)

[185] Vigeland S, Yunes N and Stein L 2011 Phys. Rev. D83 104027 (Preprint 1102.3706)

[186] Cardoso V, Pani P and Rico J 2014 Phys. Rev. D89 064007 (Preprint 1401.0528)

[187] Johannsen T 2013 Phys. Rev. D88 044002 (Preprint 1501.02809)

[188] Psaltis D 2008 Living Rev. Rel. 119 (Preprint 0806.1531)

[189] Bambi C 2015 (Preprint 1509.03884)

[190] Johannsen T 2016 Class. Quant. Grav. 33124001 (Preprint 1602.07694)

[191] Will C M 2008 Astrophys. J. 674 L25-L28 (Preprint 0711.1677)

[192] Merritt D, Alexander T, Mikkola S and Will C M 2010 Phys. Rev. D81 062002 (Preprint 0911.4718)

[193] Psaltis D, Wex N and Kramer M 2016 Astrophys. J. 818121 (Preprint 1510. 00394)

[194] Christian P, Psaltis D and Loeb A 2015 (Preprint 1511.01901)

[195] Wex N and Kopeikin S 1999 Astrophys. J. 514388 (Preprint astro-ph/9811052)

[196] Liu K, Wex N, Kramer M, Cordes J M and Lazio T J W 2012 Astrophys. J. 747 1 (Preprint 1112.2151)

[197] Novikov I D and Thorne K S 1973 Astrophysics and black holes Proceedings, Ecole d'Eté de Physique Théorique: Les Astres Occlus pp 343-550

[198] Page D N and Thorne K S 1974 The Astrophysical Journal 191 499-506

[199] Shibata M and Sasaki M 1998 Phys. Rev. D58 104011 (Preprint gr-qc/9807046) 
[200] Fabian A, Rees M, Stella L and White N E 1989 Monthly Notices of the Royal Astronomical Society 238 729-736

[201] Li L X, Zimmerman E R, Narayan R and McClintock J E 2005 Astrophys. J. Suppl. 157 335-370 (Preprint astro-ph/0411583)

[202] McClintock J E, Narayan R and Steiner J F 2014 Space Sci. Rev. 183 295-322 (Preprint 1303.1583)

[203] Reynolds C S 2014 Space Sci. Rev. 183 277-294 (Preprint 1302.3260)

[204] Johannsen T and Psaltis D 2010 Astrophys. J. 716 187-197 (Preprint 1003.3415)

[205] Johannsen T and Psaltis D 2013 Astrophys. J. 77357 (Preprint 1202.6069)

[206] Bambi C 2013 Phys. Rev. D87 023007 (Preprint 1211.2513)

[207] Jiang J, Bambi C and Steiner J F 2015 JCAP 1505025 (Preprint 1406.5677)

[208] Jiang J, Bambi C and Steiner J F 2015 Astrophys. J. 811130 (Preprint 1504.01970)

[209] Johannsen T 2014 Phys. Rev. D90 064002 (Preprint 1501.02815)

[210] Moore C J and Gair J R 2015 Phys. Rev. D92 024039 (Preprint 1507.02998)

[211] Hoormann J K, Beheshtipour B and Krawczynski H 2016 Phys. Rev. D93 044020 (Preprint 1601.02055)

[212] Bambi C and Barausse E 2011 Astrophys. J. 731121 (Preprint 1012.2007)

[213] Bambi C 2012 Astrophys. J. 761174 (Preprint 1210.5679)

[214] Kong L, Li Z and Bambi C 2014 Astrophys. J. 79778 (Preprint 1405.1508)

[215] Bambi C 2014 Phys. Rev. D90 047503 (Preprint 1408.0690)

[216] Bardeen J M, Press W H and Teukolsky S A 1972 Astrophys. J. 178347

[217] Cunningham C T 1975 The Astrophysical Journal 202 788-802

[218] Luminet J P 1979 Astronomy and Astrophysics 75 228-235

[219] Falcke H, Melia F and Agol E 2000 Astrophys. J. 528 L13 (Preprint astro-ph/ 9912263)

[220] Gillessen S, Eisenhauer F, Trippe S, Alexander T, Genzel R, Martins F and Ott T 2009 Astrophys. J. 692 1075-1109 (Preprint 0810.4674)

[221] Reid M J et al. 2014 Astrophys. J. 783130 (Preprint 1401.5377)

[222] Chatzopoulos S, Fritz T K, Gerhard O, Gillessen S, Wegg C, Genzel R and Pfuhl O 2015 MNRAS 447 948-968 (Preprint 1403.5266)

[223] Weinberg N N, Milosavljevic M and Ghez A M 2005 Astrophys. J. 622878 (Preprint astro-ph/0404407)

[224] Johannsen T, Broderick A E, Plewa P M, Chatzopoulos S, Doeleman S S, Eisenhauer F, Fish V L, Genzel R, Gerhard O and Johnson M D 2016 Phys. Rev. Lett. 116031101 (Preprint 1512.02640)

[225] Doeleman S et al. 2008 Nature 45578 (Preprint 0809.2442) 
[226] Pani P and Cardoso V 2009 Phys. Rev. D79 084031 (Preprint 0902.1569)

[227] Cunha P V P, Herdeiro C A R, Radu E and Runarsson H F 2015 Phys. Rev. Lett. 115211102 (Preprint 1509.00021)

[228] Quevedo H 1990 Fortschritte der Physik/Progress of Physics 38 733-840

[229] Sotiriou T P and Apostolatos T A 2004 Class. Quant. Grav. 21 5727-5733 (Preprint $\mathrm{gr}-\mathrm{qc} / 0407064)$

[230] Hoenselaers C and Perjés Z 1990 Classical and Quantum Gravity 71819

[231] Bergmann P G 1968 Int. J. Theor. Phys. 1 25-36

[232] Wagoner R V 1970 Phys. Rev. D1 3209-3216

[233] Blanchet L and Damour T 1986 Philosophical Transactions of the Royal Society of London A: Mathematical, Physical and Engineering Sciences 320 379-430 